\title{
Solving a Real-Time Allocation Problem with Constraint Programming
}

\author{
Pierre-Emmanuel Hladik ${ }^{\mathrm{a}, \mathrm{b}, *}$, Hadrien Cambazard ${ }^{\mathrm{b}}$, \\ Anne-Marie Déplanche ${ }^{\mathrm{a}}$, Narendra Jussien ${ }^{\mathrm{b}}$ \\ ${ }^{a}$ Université de Nantes, IRCCyN, UMR CNRS 659, 1 rue de la Noë-BP 9210, \\ 44321 Nantes Cedex 3, France \\ ${ }^{\mathrm{b}}$ École des Mines de Nantes, LINA CNRS, 4 rue Alfred Kastler - BP 20722, \\ 44307 Nantes Cedex 3, France
}

\begin{abstract}
In this paper, we present an original approach (CPRTA for "Constraint Programming for solving Real-Time Allocation") based on constraint programming to solve a static allocation problem of hard real-time tasks. This problem consists in assigning periodic tasks to distributed processors in the context of fixed priority preemptive scheduling. CPRTA is built on dynamic constraint programming together with a learning method to find a feasible processor allocation under constraints. Two efficient new approaches are proposed and validated with experimental results. Moreover, CPRTA exhibits very interesting properties. It is complete (if a problem has no solution, the algorithm is able to prove it); it is non-parametric (it does not require specific tuning) thus allowing a large diversity of models to be easily considered. Finally, thanks to its capacity to explain failures, it offers attractive perspectives for guiding the architectural design process.
\end{abstract}

Key words: hard real-time task allocation, fixed priority scheduling, constraint programming, global constraint, Benders decomposition

\section{Introduction}

Real-time systems have applications in many industrial areas: telecommunication systems, the automotive and aircraft industries, robotics, etc. Today's applications are becoming more and more complex, not only in their software (an

* Corresponding author. Tel: 00 33(0)251-8582-25 Fax: 00 33(0)251-8582-49

Email address: Pierre-Emmanuel.Hladik@emn.fr (Pierre-Emmanuel Hladik). 
increasing number of concurrent tasks with various interaction schemes) and their execution platform (many distributed processing units interconnected through specialized network(s)), but also in their numerous functional and non-functional requirements (timing, resources, power, etc.). One of the main issues in the architectural design of such complex distributed applications is to define an allocation of tasks onto processors so as to meet all the specified requirements. Even if it has to be solved off-line most of the time, it needs efficient and adaptable search techniques, which can be integrated into a more global design process. Furthermore, it is desirable that these techniques return relevant information intended to help the designer who is faced with architectural choices. In particular the "binary" result (has a feasible allocation been found? Yes and here it is, or no, and that is all) which is usually returned by the search algorithm is not satisfactory in failure situations. The designer expects some explanations justifying the failure and enabling him to revisit his design. Therefore, more sophisticated search techniques that can collect some knowledge about the problem they solve are required. These are the general objectives of the work we are conducting.

More precisely, the problem we are concerned with consists in assigning a set of periodic, pre-emptive tasks to distributed processors in the context of fixed priority scheduling not only to respect schedulability but also to account for requirements related to memory capacity, co-residence, redundancy, and so on. The mapping we are concerned with is static, i.e. all allocation decisions are made off-line, before the application runs, and they do not change afterwards. We assume that the characteristics of tasks (execution time, priority, etc.) and those of the physical architecture (processors and network) are all known $a$ priori.

In the literature, the allocation problem is studied extensively. A classification of related works is difficult because of the varied nature of the hardware architecture model (multiprocessor, distributed, homogeneous, etc.), the software architecture model (periodic tasks, deadline, precedence relations, etc.), the set of constraints (memory, allocation constraints, etc.), the objective (to minimize execution and communication costs, to balance the load, etc.), and the solving strategy.

Since the problem of allocating tasks is generally NP-hard [34], some form of enumerative method or approximation using heuristics needs to be developed for this problem: graph theory techniques $[57,38,18]$, branch-andbound $[41,45,44,50,65]$, genetic algorithm $[40,6,53,2,39,19,20,42,21]$, clustering $[4,1,36]$, steepest descent (or Hill climbing) [40,64], tabu search [46,64]; simulated annealing $[63,11,40,14,15,17,19,64]$, neural network $[56,2]$, and dedicated heuristics $[54,17,32,66,3]$. However, today, for a specific problem, no technique seems to be more appropriate than another. 
Moreover, the majority of these techniques suffer from two main drawbacks: firstly, their implementation is widely conducted by the considered models or objective, so it is difficult to add a new constraint or to consider a new model; secondly, their performances are sensitive to initial parameters and need experimental tuning.

That is why we decided to tackle the allocation problem with a quite innovative approach, constraint programming (cp). The main advantages of $\mathrm{cp}$ are: its declarativity, the variables, domains, constraints are simply described; its genericity, it is not a problem-dependent technique, general rules are mechanically performed during the search; its adaptability, each constraint can be considered as independent and a model could be simply extended by merging these different constraints; and its non-parametric ability. This technique has been widely used to solve a large range of combinatorial problems. It has proved quite effective in a variety of applications (from planning and scheduling to finance - portfolio optimization - through biology). Up to now, solving the allocation problem of hard real-time tasks with cp has not been studied extensively. To our knowledge, only Szymanek et al. [58], Schild and Würtz [55] and Ekelin [16] have used cp to produce an assignment and a pre-runtime scheduling of distributed systems under optimization criteria. Even though their context is different from ours, their results have shown the ability of such an approach to solve an allocation problem for embedded systems and have encouraged us to go further.

In this paper, two approaches are considered. The first one introduces a global constraint $^{1}$ and an ad hoc algorithm, i.e. a filtering algorithm, to tackle schedulability. This algorithm is a custom-written filtering algorithm designed to take into account and to exploit the structure of the schedulability constraints. The second investigated approach uses the complementary strengths of constraint programming and optimization methods from operational research like numerous hybridation schemes $[61,28,26,8]$. It is a decompositionbased method (related to logic-based Benders decomposition [27]), which separates the allocation problem from the scheduling one: the allocation problem is solved by means of dynamic constraint programming tools, whereas the scheduling problem is treated with specific real-time schedulability analysis. The main idea is to "learn" from the schedulability analysis to re-model the allocation problem so as to reduce the search space. In that sense, we can compare this approach to a form of learning from failures.

First experimental results show that these two methods produce an efficient way to solve the allocation problem. Moreover, a fundamental property of these methods is their completeness: when a problem has no solution, they are able to prove it (contrary to heuristic methods that are unable to decide).

$\overline{1}$ A constraint is said to be global when it is a conjunction of a set of constraints. 
Moreover, they are also able to produce an explanation when they fail to find a solution. As will be shown in the paper, this explanation could be used in the future as a way to help the designer of real-time systems during the design process.

The remainder of this paper is organized as follows. In Section 2, we describe the problem. Some related works are presented in Section 3. Section 4 briefly introduces constraint programming before translating the problem as a constraint satisfaction one in Section 5. The two approaches we propose are then described: Section 6 is concerned with the global constraint and Section 7 is dedicated to the logical Benders decomposition. Some experimental results are presented in Section 8. Section 9 shows how it is possible to set up a failure analysis able to aid the designer to review his plans. It is a first attempt that proves its feasibility but it will need to go deeper. The paper ends with concluding remarks in Section 10.

\section{Description of the problem}

We base our research on the model in [63] with few differences as the communication protocol and the schedulability test.

\subsection{The real-time system architecture}

The hard real-time system we consider can be modeled by a software architecture, the set of tasks, and a hardware architecture, the execution platform for the tasks, as represented in Fig. 1.

By hardware architecture, we mean a set $\mathcal{P}=\left\{p_{1}, \ldots, p_{k}, \ldots, p_{m}\right\}$ of $m$ processors with fixed memory capacity $m_{k}$ and identical processing speed. Each processor schedules tasks assigned to it with a fixed priority strategy. It is a simple rule: a static priority is given to each task and, at run-time, the ready task with the highest priority is put in the running state, pre-empting a lower priority task. These processors are fully connected through a communication medium with a bandwidth $\delta$. In this paper, we look at a communication medium called a $C A N$ bus, which is currently used in a wide spectrum of realtime embedded systems. However, any other communication network could be considered if its timing behavior (including its protocol rules) is predictable. Thus, the first experiments we conducted addressed a token ring network [24].

CAN (Controller Area Network) [10] is both a protocol and a physical network. 
CAN works as a broadcast bus, meaning that all connected nodes can read all messages sent on the bus. Each message has a unique identifier, which is also used as the message priority. On each node, waiting messages are queued. The bus makes sure that when a new message is selected to transfer, the message with the highest priority, waiting on any connected node, will be transmitted first. When at least one bit of a message has started to be transferred it cannot be pre-empted even though higher priority messages arrive. As a result, the CAN behavior will be seen subsequently as that of a non-pre-emptive fixed priority message scheduling.

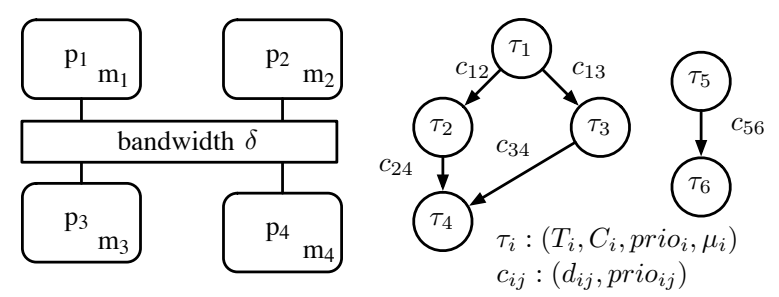

Fig. 1. An example of hardware (left) and software (right) architecture.

The software architecture is modeled as a valued, oriented and acyclic graph $(\mathcal{T}, \mathcal{C})$. The set of nodes $\mathcal{T}=\left\{\tau_{1}, \ldots, \tau_{n}\right\}$ represents the tasks. A task in turn is a set of instructions that must be executed sequentially in the same processor. The set of edges $\mathcal{C} \subseteq \mathcal{T} \times \mathcal{T}$ refers to the data sent between tasks.

A task $\tau_{i}$ is defined through timing characteristics and resource needs: its period $T_{i}$ (as a task is periodically activated, the date of its first activation is free), its worst-case execution time without pre-emption $C_{i}$ and its memory need $\mu_{i}$. A priority prio $_{i}$ is given to each task. Task $\tau_{j}$ has priority over $\tau_{i}$ if, and only if, prio $_{i}<$ prio $_{j}$. In our approach, we treat allocation as an issue separate from that of scheduling. Priority assignment is globally performed before allocation. Rate monotonic or deadline monotonic algorithms can be used. However, our approach does not consider a specific scheduling policy.

Edges $c_{i j}=\left(\tau_{i}, \tau_{j}\right) \in \mathcal{C}$ are weighted with the amount of exchanged data $d_{i j}$ together with a priority value prio $_{i j}$ (useful in the CAN context) ${ }^{2}$. In this model, we assume that communicating tasks have the same activation period but we do not consider any precedence constraint between them. They are periodically activated in an independent way, and they read input data and write output data at the beginning and the end of their execution.

The underlying communication model is inspired by OSEK-COM specifications [43]. OSEK-COM is a uniform communication environment for automo-

$\overline{2}$ Task priorities are all assumed to be different. The same assumption is made about message priorities. 


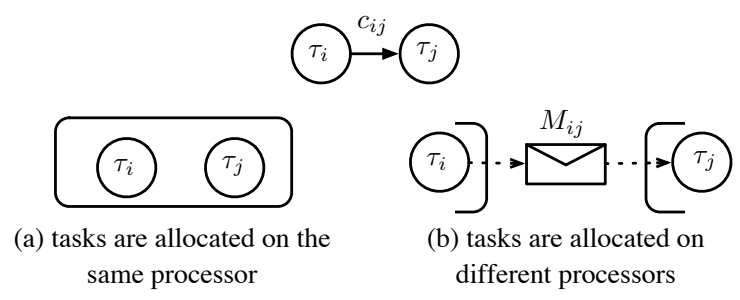

Fig. 2. Depending on the task allocation, a message exists or not.

tive control unit application software. It defines common software communication interface and behavior for internal communications (within an electronic control unit) and external ones (between networked vehicle nodes) which are independent of the communication protocol used. It is the following. Tasks that are located on the same processor communicate through local memory sharing. Such a local communication cost is assumed to be zero. On the other hand, when two communicating tasks are assigned to two distinct processors, the data exchange needs the transmission of a message on the network. Here we are interested in the periodic transmission mode of OSEK-COM. In this mode, data production and message transmission are not synchronized: a producer task writes its output data into a local unqueued buffer from where a periodic protocol service reads it and sends it into a message. The building of protocol data units considered here is very simple: each piece of data $d_{i j}$ that has to be sent from a producer task $\tau_{i}$ to a consumer task $\tau_{j}$ in a distant way gives rise to its own message $M_{i j}$. Moreover, in this paper, for the sake of simplicity, the asynchronous receiving mode is preferred. It means that the release of a consumer task $\tau_{j}$ is strictly periodic and unrelated to the $M_{i j}$ message arrival. Thus, when a node receives a message from the bus, its protocol records its data into a local unqueued buffer from where it can be read by the task $\tau_{j}$. In [25], an extension of this work to a synchronous receiving mode is proposed in which a message reception notification activates the consumer task.

As a result, depending on the task allocation, an edge $c_{i j}$ of the software architecture may give rise to two different equivalent schemes as illustrated in Fig. 2. In Fig. 2(b), $M_{i j}$ inherits its period $T_{i}$ from $\tau_{i}$ and its priority prio ${ }_{i j}$ from $c_{i j}$.

Therefore, from a scheduling point of view, messages on the bus are very similar to tasks on a processor. Like for tasks, each message $M_{i j}$ is "activated" every $T_{i}$ units of time; its (bus) priority is prio $_{i j}$; and it has a transmission time $C_{i j}$ (the time it takes to transfer the message on the bus, see Section 2.2.3 for its computation). 


\subsection{The allocation problem}

An allocation is a mapping $A: \mathcal{T} \rightarrow \mathcal{P}$ such that:

$$
\tau_{i} \mapsto A\left(\tau_{i}\right)=p_{k}
$$

The allocation problem consists in finding a mapping $A$ that respects the whole set of constraints described below.

\subsubsection{Resource constraints}

Three kinds of constraints are considered ${ }^{3}$ :

- Memory capacity: The memory use of a processor $p_{k}$ cannot exceed its capacity $\left(m_{k}\right)$ :

$$
\forall k=1 . . m, \sum_{A\left(\tau_{i}\right)=p_{k}} \mu_{i} \leq m_{k}
$$

- Utilization factor: The utilization factor of a processor cannot exceed its processing capacity. The following inequality is a necessary schedulability condition:

$$
\forall k=1 . . m, \sum_{A\left(\tau_{i}\right)=p_{k}} \frac{C_{i}}{T_{i}} \leq 1
$$

- Network use: To avoid overload, the messages carried along the network per unit of time cannot exceed the network capacity:

$$
\sum_{\substack{c_{i j}=\left(\tau_{i}, \tau_{j}\right) \\ A\left(\tau_{i}\right) \neq A\left(\tau_{j}\right)}} \frac{s_{i j}}{T_{i}} \leq \delta
$$

where $s_{i j}$ is the message size; it is a function of $d_{i j}$ depending on the message structure (see Section 2.2.3).

\subsubsection{Allocation constraints}

Allocation constraints are due to the system architecture. We distinguish three kinds of constraint.

- Residence: a task may need a specific hardware or software resource that is only available on specific processors (e.g. a task monitoring a sensor has to run on a processor connected to the input peripheral). This constraint is

$\overline{3}$ Precise units are not specified but obviously they have to be consistent with the given expressions. 
expressed as a couple $\left(\tau_{i}, \alpha\right)$ where $\tau_{i} \in \mathcal{T}$ is a task and $\alpha \subseteq \mathcal{P}$ is the set of available host processors for the task. A given allocation $A$ must respect:

$$
A\left(\tau_{i}\right) \in \alpha
$$

- Co-residence: several tasks may have to be assigned to the same processor (they share a common resource). Such a constraint is defined by a set of tasks $\beta \subseteq \mathcal{T}$ and any allocation $A$ has to fulfil:

$$
\forall\left(\tau_{i}, \tau_{j}\right) \in \beta^{2}, A\left(\tau_{i}\right)=A\left(\tau_{j}\right)
$$

- Exclusion: Some tasks may be replicated for some fault-tolerance objectives and therefore cannot be assigned to the same processor. It corresponds to a set $\gamma \subseteq \mathcal{T}$ of tasks, which cannot be put together. An allocation $A$ must satisfy:

$$
\forall\left(\tau_{i}, \tau_{j}\right) \in \gamma^{2}, A\left(\tau_{i}\right) \neq A\left(\tau_{j}\right)
$$

\subsubsection{Timing constraints}

Timing constraints are expressed by means of the relative deadlines for the tasks and messages. A timing constraint enforces the duration between the activation date of any instance of the task $\tau_{i}$ and its completion time to be bounded by its relative deadline $D_{i}$. Depending on the task allocation, such timing constraints may concern the instantiated messages too. For tasks as well as messages, their relative deadline is hereafter assumed equal to their activation period.

A widely chosen approach for the schedulability analysis of a task and message set $S$ is based on the following necessary and sufficient condition [35]: $S$ is schedulable if, and only if, for each task and message of $S$, its worst-case response time is less than or equal to its relative deadline. Thus, it leads us to compute worst-case response times for the tasks on the processors and for the messages on the bus. According to the features of the considered task and message models, as well as the processor and bus scheduling algorithms, a classical computation can be used and its main results are given below.

Task worst-case response time. For independent and periodic tasks with a pre-emptive fixed priority scheduling algorithm, it has been proved that the worst-case execution scenario for a task $\tau_{i}$ happens when it is released simultaneously with all the tasks that have a priority higher than prio $_{i}$. When $D_{i}$ is (less or) equal to $T_{i}$, the worst-case response time for $\tau_{i}$ is given by [35]:

$$
R_{i}=C_{i}+\sum_{\tau_{j} \in h p_{i}(A)}\left\lceil\frac{R_{i}}{T_{j}}\right\rceil C_{j}
$$


where $\lceil x\rceil$ is the smallest integer greater than $x$ and $h p_{i}(A)$ is the set of tasks with a priority higher than prio $_{i}$ and located on the processor $A\left(\tau_{i}\right)$ for a given allocation $A$. The summation gives the time that tasks with higher priority will take before $\tau_{i}$ has completed. The worst-case response time $R_{i}$ can be easily solved by looking for the fix-point of Eq. (8) in an iterative way.

Message worst-case response time. As mentioned earlier, message scheduling on the CAN bus can be viewed as a non-pre-emptive fixed priority scheduling strategy. Thus, when writing the worst-case response time equation for a message, Eq. (8) has to be reused with some modifications. First, it has to be changed so that a message can only be pre-empted during its first transmitted bit instead of its whole execution time. Second, a blocking time, i.e. the longest time the message might be blocked by a lower priority message, must be added. The resulting worst-case response time equation for the CAN message $M_{i j}$ is [62]:

$$
R_{i j}=C_{i j}+L_{i j}
$$

with

$$
L_{i j}=\sum_{M^{\prime} \in h p_{i j}(A)}\left\lceil\frac{L_{i j}+\tau_{b i t}}{T^{\prime}}\right\rceil C^{\prime}+\max _{M^{\prime} \in l p_{i j}(A)}\left\{C^{\prime}-\tau_{b i t}\right\}
$$

where $h p_{i j}(A)$ (respectively $l p_{i j}(A)$ ) is the set of messages derived from the allocation $A$ with a priority higher (respectively lower) than prio $_{i j} ; \tau_{b i t}$ is the transmission time for one bit ( $\tau_{b i t}$ is related to the bus bandwidth $\delta, \tau_{b i t}$ (second) $=1 / \delta$ (bit per second) $) ; C^{\prime}$ is the worst-case transmission time for the message $M^{\prime}$.

Here as well the computation of Eq. (10) can be solved iteratively.

To calculate the worst-case transmission time $C_{i j}$ of a message, Eq. (11) is used [62]:

with

$$
C_{i j}=s_{i j} \tau_{b i t}
$$

$$
s_{i j}=\left\lfloor\frac{34+8 d_{i j}}{5}\right\rfloor+47+8 d_{i j}
$$

It shows that the message size is not given directly from the data size $d_{i j}$ (in bytes) and the frame overhead of 47 bits (identifier, CRC, etc.). It has to take into account the possible overhead caused by the bit stuffing process of CAN controllers.

From now on, an allocation $A$ is said to be valid if it meets allocation and resource constraints. It is schedulable if it meets timing constraints. Finally, a solution to our problem is a valid and schedulable allocation of the tasks.

Notice that the objective of this article is to validate an application (find a solution) and not optimize a function such as workload balancing $[60,2]$, the 
number processors used [42] nor the response time of tasks [45,2].

\section{Related work}

In a general way, the task mapping problem includes assigning (allocation) each task to a processor and ordering (scheduling) the execution of the tasks on each one such that all functional and temporal constraints of the system are respected. In the literature, mapping problems are studied extensively and an exhaustive overview, or even a classification of them, is difficult because of the huge variety of problems and solving techniques they consider.

For models quite similar to the one presented in Section 2, [63,11,41,54,4,50,21] are good examples of the research efforts. Like us, they all consider a fixed priority scheduler. Allocation and scheduling are usually considered as two independent stages. For most of them [63,11,41,54,4,21], only the allocation problem is solved since task priorities are known. In [50], Richard et al. propose a method that simultaneously allocates tasks to processors and assigns priorities to tasks and messages.

Different techniques are used: a simulated annealing algorithm in [63,11], a branch-and-bound search algorithm in [41,50], a genetic algorithm in [21], and a dedicated heuristic in [54]. In [4], a clustering algorithm is proposed. In that case, the solving process is divided into two steps. Firstly, the search space is reduced by merging some tasks according to some specific criteria (this is the clustering step). Secondly, a classic solving algorithm (simulated annealing, branch-and-bound, etc.) is used for allocating task clusters to processors. However, by reducing the search space, clustering suffers from the risk of not finding any feasible assignment.

All these techniques seem effective ways for solving allocation problems. However, they all suffer from two important drawbacks: 1) they are sensitive to their initial parameters, i.e. temperature and cooling for simulated annealing, selection criteria and population size for genetic algorithm, enumeration order for branch-and-bound, etc. 2) much work is necessary to efficiently extend a method to a new model, i.e. the objective function of the simulated annealing must be redefined, branch-and-bound functions have to be designed for each mapping problem, crossing-over or mutation operators of genetic algorithm are dedicated to only one problem, etc.

Now it seemed to us that a constraint programming (cp) approach should be able to limit, indeed to avoid, these disadvantages. Moreover, it constitutes a new solving strategy for the real-time task allocation problem. It is true that little attention has been paid to it $[55,58,59,16]$. Furthermore, these dif- 
fer fundamentally in the scheduling technique: only cyclic task schedulers are assumed, i.e. a scheduling cycle is derived before runtime, and the runtime system dispatches tasks based on current time in respect with the cycle. The fact that only a cyclic scheduling is considered makes the problem of mapping very different from ours. Indeed, the scheduling cycle is viewed as a set of discrete intervals, the constraints of which can be naturally expressed in the classical formalism of $\mathrm{cp}$. The main problem with dynamic scheduling is that it is not possible to simply express the schedulability test as an efficient constraint for a cp solver.

In [55], precedence constraints between tasks as well as end-to-end deadlines are taken into account for computing the scheduling cycle. In [58], more constraints are considered: i) resources (memory and shared resource), ii) communications (a cyclic scheduling is also produced for messages), iii) power consumption. In [16], Ekelin summarizes of all these works and proposes a general approach for multi-constraints and multi-objective functions for realtime cyclic scheduling. As in [4], Szymanek and Kuchcinski [59] studied the problem of task clustering under multi-resource constraints.

The first objective of our work has been to study the applicability of cp to our allocation problem. It has led us to propose an efficient way to include the dynamic scheduling model in the cp solver. From this point of view, this paper makes an innovative contribution in comparison with previous works.

\section{A short introduction to constraint programming}

A constraint satisfaction problem (csp) consists of a set $V$ of variables $x_{i}$ defined by a corresponding set $D_{i}$ of possible values (the so-called domain) and a set $C$ of constraints. A solution to the problem is an assignment of a value in $D_{i}$ to each variable $x_{i}$ in $V$ such that all constraints are satisfied. For example, consider a 3-uple of variables $V=\left\{x_{1}, x_{2}, x_{3}\right\}$, their domains $D_{1}=D_{2}=D_{3}=\{1,2,3\}$ and two constraints $C_{1}: x_{1}>x_{2}$ and $C_{2}: x_{1}=x_{3}$. A solution of this csp is $x_{1}=2, x_{2}=1$ and $x_{3}=2$.

The solutions to a csp can be found by systematically searching through the possible assignments of values to variables. The variables are sequentially labeled and, as soon as all the variables relevant to a constraint are instantiated, the validity of the constraint is checked. If any of the constraints is violated, backtracking is performed to the most recently instantiated variable that still has available values.

However, cp offers more accurate methods to solve a csp. One of them is based on removing inconsistent values from domains of variables till a solution is 
found. Consider the previous example: the value 1 could be removed from $D_{1}$, because $C_{1}$ cannot be respected if $x_{1}=1$. Several consistency techniques exist and they are combined to solve a csp. For example, when a value is removed from the domains of variables, it could be propagated through other constraints. In the previous example, after removing 1 from $D_{1}, 1$ could be removed from $D_{3}$ because of $C_{2}$.

This mechanism coupled with a backtracking scheme allows the search space to be explored in a complete way. For a more detailed introduction to $\mathrm{cp}$, we refer the reader to [52].

In this paper, the search is performed by the Maintaining Arc-Consistency algorithm (mac). mac is nowadays considered as one of the best algorithms for solving a csp. Moreover, a specific mac algorithm has been used, based on the use of explanations. In a few words, an explanation can be considered as a limited trace of the past activity of the constraint solver. It is a set of constraints, and records enough information to justify any decision of the solver such as a reduction of domain or a contradiction. Due to space limitation, we cannot expand upon this point but the reader may refer to [30] for a detailed presentation.

\section{Translation of the allocation problem into a csp}

The first thing one has to do when using $\mathrm{cp}$ to solve a problem is to word it as a csp. In our case, it relies on a redundant formulation using three sets of variables: $x, y, w$.

Let us first consider $n$ integer-valued variables $x_{i}$ which are decision variables, each one corresponding to one task, and representing the processor selected to process the task: $\forall i \in\{1 . . n\}, x_{i} \in\{1, \ldots, m\}$. Then, boolean variables $y_{i p}$ indicate the presence of a task on a processor: $\forall i \in\{1 . . n\}, \forall p \in$ $\{1 . . m\}, y_{i p} \in\{0,1\}$. Finally, boolean variables $w_{i j}$ are introduced to express whether a pair of tasks exchanging data are located on the same processor or not: $\forall c_{i j}=\left(\tau_{i}, \tau_{j}\right) \in \mathcal{C}, w_{i j} \in\{0,1\}$. Integrity constraints are used to enforce the consistency of the redundant model. This redundant model has been chosen to speed up the search and propagate algorithms.

Moreover, the constraints of our allocation problem have to be mapped on this model. It appears that allocation and resource constraints can be directly expressed with the classical constraints of $\mathrm{cp}$. Their translation into the csp are given here:

\section{- Resource constraints}


- Memory capacity: (cf. Eq. (2)) $\forall p \in\{1 . . m\}, \sum_{i \in\{1 . . n\}} y_{i p} \mu_{i} \leq \mu_{p}$

- Utilization factor: ( $c f$. Eq. (3)) Let $\operatorname{lcm}(T)$ be the least common multiple of periods of the tasks ${ }^{4}$. The constraint can be written as follows:

$$
\forall p \in\{1 . . m\}, \quad \sum_{i \in\{1 . . n\}} \frac{y_{i p} \operatorname{lcm}(T) C_{i}}{T_{i}} \leq \operatorname{lcm}(T)
$$

- Network use: ( $c f$. Eq. (4)) The network capacity is bound by $\delta$. Therefore, the size of the set of messages carried on the network cannot exceed this limit:

$$
\sum_{i \in\{1 . . n\} j \in\{1 . . n\}} \frac{w_{i j} \operatorname{lcm}(T) s_{i j}}{T_{i}} \leq \operatorname{lcm}(T) \delta
$$

\section{- Allocation constraints}

- Residence: (cf. Eq. (5)) it consists of forbidden values for $x$. A constraint is added for each forbidden processor $p$ of $\tau_{i}: x_{i} \neq p$

- Co-residence: (cf. Eq. (6)) $\forall\left(\tau_{i}, \tau_{j}\right) \in \beta^{2}, x_{i}=x_{j}$

- Exclusion ${ }^{5}$ : (cf. Eq. (7)) AllDifferent $\left(x_{i} \mid \tau_{i} \in \gamma\right)$

However, because of the schedulability analysis they require, timing constraints cannot be translated as directly as the previous ones. Two approaches have been studied to consider timing constraints. For the first approach, timing constraints are taken into account at each variable assignment the solver makes. Partial schedulability analyses must be conducted and their results transformed into new constraints on variables. This method is introduced in Section 6. Inversely, the second approach consists in breaking down the allocation problem into two subproblems (one that deals with allocation and resource constraints, the other with timing constraints), and managing cooperation between them so as to find a valid and schedulable solution. Section 7 is dedicated to this method.

\section{A global constraint for schedulability}

To tackle schedulability during search, a classical approach is to integrate it as a constraint of the cp solver. For this purpose, some notations used previously for a full allocation are extended to a partial one:

4 Utilization factor and network use are reformulated with the lcm of task periods because our constraint solver cannot currently handle constraints with both real coefficients and integer variables.

5 An AllDifferent constraint on a set $V$ of variables ensures that all variables among $V$ have different values. 
Definition 6.1 A partial allocation is a mapping $a: \mathcal{U} \subset \mathcal{T} \rightarrow \mathcal{P}$ such that:

$$
\tau_{i} \in \mathcal{U} \mapsto a\left(\tau_{i}\right)=p_{k}
$$

Definition 6.2 We call a decision from a partial allocation $a: \mathcal{U} \rightarrow \mathcal{P}$, a partial allocation $\delta$ of a task set $\Delta \subsetneq \mathcal{U}$. We denote the new allocation produced as $a^{\prime}=a+\delta$. It is such that $a^{\prime}: \mathcal{U} \cup \Delta \rightarrow \mathcal{P}$ and $\forall \tau_{i} \in \mathcal{U}, a^{\prime}\left(\tau_{i}\right)=a\left(\tau_{i}\right)$, $\forall \tau_{i} \in \Delta, a^{\prime}\left(\tau_{i}\right)=\delta\left(\tau_{i}\right)$

We denote $R_{i}(a)$ the worst-case response time of $\tau_{i}$ for a partial allocation $a$. It is the worst-case response time that $\tau_{i}$ would exhibit if only those tasks allocated in $a$ were considered. $h p_{i}(a)$, respectively $l p_{i}(a)$ is the set of higher, respectively lower, priority tasks than $\tau_{i}$ on the same processor as $a\left(\tau_{i}\right)$.

\subsection{Filtering algorithm}

The cp solver proceeds step by step. At each step, a decision is taken from a partial allocation and a filtering algorithm is used. A filtering algorithm associated with a constraint $C$ is an algorithm that may remove some values that are inconsistent with $C$, but that does not remove any consistent values [49]. For example, consider the same csp as in Section 4: a 3-uple of variables $\left\{x_{1}, x_{2}, x_{3}\right\}$, their domains $D_{1}=D_{2}=D_{3}=\{1,2,3\}$ and two constraints $C_{1}: x_{1}<x_{2}$ and $C_{2}: x_{1}=x_{3}$. In the first step, $x_{1}$ takes the value 1. The filtering algorithm of $C_{1}$ removes the value 1 of $D_{2}$ and the filtering algorithm of $C_{2}$ removes the values 2 and 3 of $D_{3}$. At the next step, $x_{2}$ takes the value 2 and so on.

The holy grail with a global constraint is to achieve generalized arc consistency (gac) which simply means a complete filtering algorithm so that any value that does not belong to a solution is eliminated. This involves providing polynomial necessary and sufficient conditions regarding the existence of a solution for the constraint (according to current domains of variables).

Table 1

\begin{tabular}{|c|c|c|c|c|c|}
\hline$\tau_{i}$ & $\tau_{1}$ & $\tau_{2}$ & $\tau_{3}$ & $\tau_{4}$ & $\tau_{5}$ \\
\hline$T_{i}$ & 20 & 10 & 15 & 15 & 4 \\
\hline$C_{i}$ & 12 & 2 & 5 & 7 & 2 \\
\hline prio $_{i}$ & 1 & 2 & 3 & 4 & 5 \\
\hline
\end{tabular}

Task and message characteristics

For example, consider a simple example of five tasks $\tau_{i}$ with domain $\left\{p_{1}, p_{2}\right\}$ such that every triple (10 triples exist) is unschedulable (Table 1 gives an example). Assigning five tasks to two processors will force placing three of them together, which will raise a contradiction as any triple is forbidden. A polynomial filtering algorithm for our global schedulability constraint that 
respects the gac should detect this inconsistency. However, we can prove it is impossible to do.

Property 6.1 Achieving generalized arc-consistency for the schedulability constraint is NP-hard.

Proof. In [37], Leung and Whitehead proved that (Theorem 2.6, page 242) for a given software architecture $\mathcal{T}$ and a hardware architecture $\mathcal{P}$, the problem of deciding whether an allocation from $\mathcal{T}$ to $\mathcal{P}$ is schedulable is NP-hard. The proof is done by reducing the 3-PARTITION problem to the schedulability problem. The 3-PARTITION is a classical problem that has been shown to be NP-complete and so achieving generalized arc-consistency for the schedulability problem is NP-hard. Moreover, as the schedulability test (Equation 8) is pseudo-polynomial, we cannot conclude whether the problem of gac is NPcomplete.

Our filtering algorithm is therefore based on a relaxation of the gac. Algorithm 1 shows the pseudo-code of the filtering algorithm for the global constraint to tackle schedulability. It begins to check the schedulability of the current partial allocation (line 1). It is easily done by computing the worstcase response time of only allocated tasks and present messages (property 11.1, Appendix 1). A present message is a message that we are sure exists for a partial allocation: consider a communication $c_{i j}$, the message $M_{i j}$ exists if, and only if, the domains of values of $\tau_{i}$ and $\tau_{j}$ are disjoint $\left(D_{i} \cap D_{j}=\emptyset\right)$. Notice that a message can exist even if its producer or its consumer is not allocated. If the allocation is schedulable, the filtering algorithm removes values that are inconsistent (lines 2 to 13) by testing the schedulability for each value of each remaining variable (line 7). Then, the pruning is propagated within the constraint set until a fix-point is reached (while loop).

This filtering can, however, miss powerful deductions of the gac. With the example presented in Table 1, an inconsistent state will not be detected by our pragmatic approach and a search tree will be built over three tasks among the five to prove this inconsistency, see Figure 3: (1) $\tau_{1}$ is allocated to $p_{1}$. All other tasks could still be allocated to $p_{1}$ (loop from line 4 to line 12). (2) $\tau_{2}$ is also allocated to $p_{1}$. The inconsistency is detected because $D_{3}, D_{4}$ and $D_{5}$ are reduced to $p_{2}$ (line 8 ) and $\tau_{3}, \tau_{4}$ and $\tau_{5}$ are not schedulable on $p_{2}$. (3) $\tau_{2}$ is allocated to $p_{2}$. All domains of unallocated tasks are still $\left\{p_{1}, p_{2}\right\}$. (4) $\tau_{3}$ is allocated to $p_{1}$. The inconsistency is detected because $D_{4}$ and $D_{5}$ are reduced to $p_{2}$ and $\tau_{2}, \tau_{4}$ and $\tau_{5}$ are not schedulable on $p_{2}$. (5) $\tau_{3}$ is allocated to $p_{2}$. The inconsistency is detected because $D_{4}$ and $D_{5}$ are reduced to $p_{1}$ and $\tau_{1}, \tau_{4}$ and $\tau_{5}$ are not schedulable on $p_{1}$. (...) and so on. However, this approach is already very costly and we will now discuss ways to speed it up. 

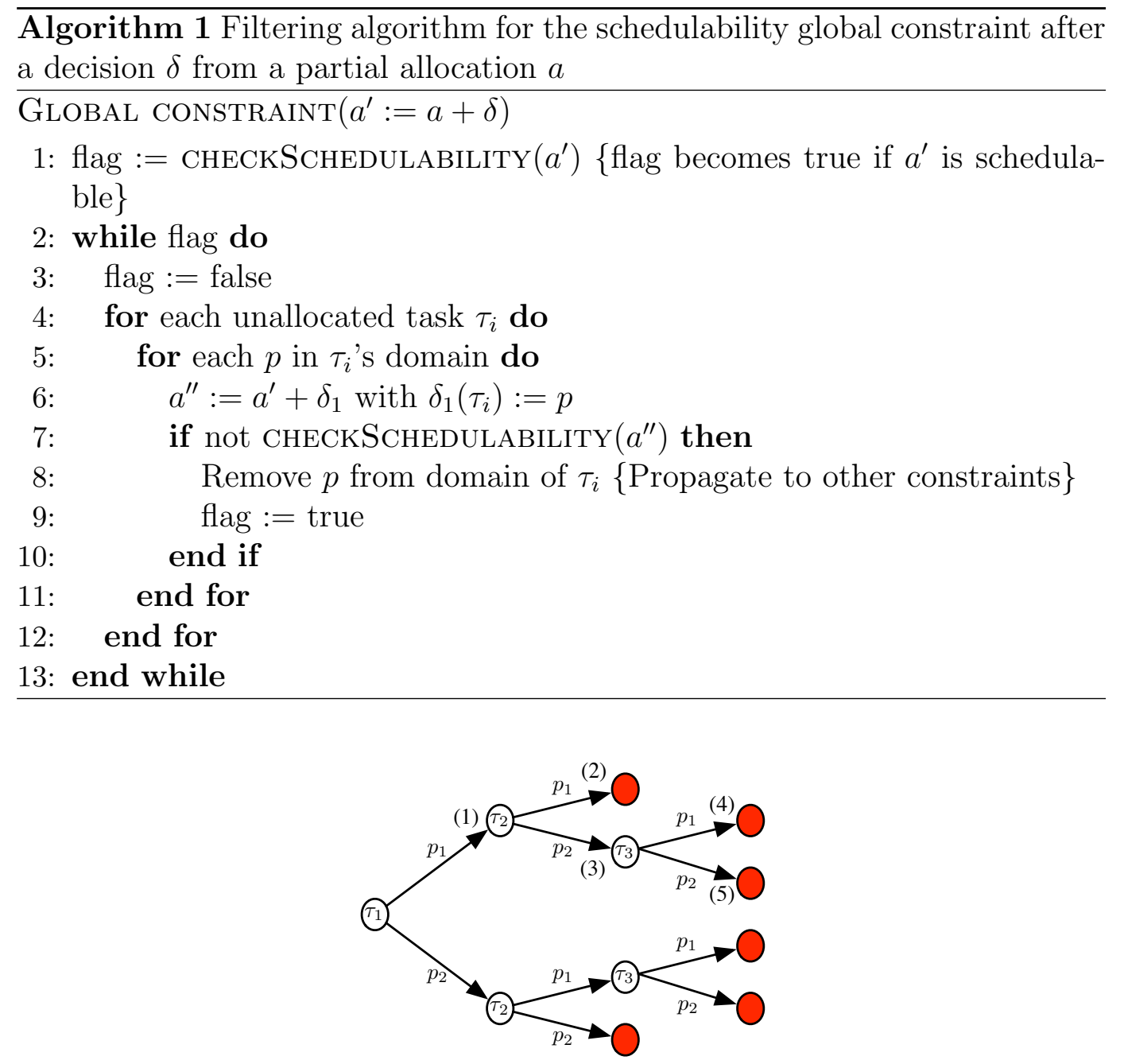

Fig. 3. Tree-search for an allocation with the global constraint. Colored nodes are those where the problem is inconsistent.

\subsection{Incrementality}

To speed up the filtering of the global constraint for a partial allocation, it is possible to use some knowledge from the previous partial allocations. This incrementality can be used twice in the filtering algorithm. Firstly, during the schedulability test and, secondly, during the propagation by reducing the number of values to check in the domains of variables. These two points are now developed.

\subsubsection{Incrementality of the schedulability test}

From now on, we suppose that all other constraints are met, and so the partial allocation is valid too. Consider a partial but schedulable allocation $a$ and a 
decision from $a$ that allocates only one task $\tau_{i}$ with $a^{\prime}\left(\tau_{i}\right)=p_{k}$. Since the scheduling of processors is made in an independent way, only those tasks on $p_{k}$ may suffer from the allocation of $\tau_{i}$. Moreover, all tasks in $h p_{i}\left(a^{\prime}\right)$ are still schedulable because the worst-case response time equation (Eq. 8) ensures that all tasks $\tau_{j} \in h p_{i}\left(a^{\prime}\right)$ keep the same response time: $R_{j}\left(a^{\prime}\right)=R_{j}(a)$.

Rule 1 When a task is allocated to a processor from a schedulable partial allocation, schedulability has to be checked only for this task and the lower priority ones on that processor.

This rule could be extended to a decision $\delta$ with $\# \Delta>1$, where $\# X$ stands for the cardinality of the set $X$. For the same reason, schedulability has to be checked only for the tasks $\tau_{i} \in \Delta, a^{\prime}\left(\tau_{i}\right)=p_{k}$, and the tasks $\tau_{j} \in \mathcal{U}$ such that $a^{\prime}\left(\tau_{j}\right)=p_{k} \wedge$ prio $_{i}>$ prio $_{j}$.

The same reasoning can be applied to message schedulability analysis.

Rule 2 When a new message appears $M_{a b}$, message schedulability has to be checked only for that message, the lower priority ones and the higher priority ones $M_{i j}$ such that $C_{a b}>\max _{M^{\prime} \in l p_{i j}(a)}\left\{C^{\prime}\right\}$.

Rule 2 can be extended to a decision that gives rise to many messages. Let $\Delta^{*}$ be the set of these new messages. After a decision $\delta$, message schedulability has to be checked only for $M_{a b}$, the highest priority message of $\Delta^{*}$, the messages in $l p_{a b}\left(a^{\prime}\right)$ and the higher priority messages $M_{i j} \in h p_{a b}(a)$ such that $\max _{M \in \Delta^{*}} C>\max _{M^{\prime} \in l p_{i j}(a)}\left\{C^{\prime}\right\}$.

These rules can be implemented into the CHECKSCHEDULABILITY function called at lines 1 and 7 in algorithm 1 .

\subsubsection{Incrementality of domains}

By considering the previous partial allocation and the current decision, the number of values in the domain of a variable that have to be checked in order to remove inconsistency values (line 5 in algorithm 1) can be reduced.

Let $a$ be a partial allocation and $\delta$ a decision such that $a^{\prime}=a+\delta, \Delta^{*}=\emptyset$ and $\tau_{i} \notin \mathcal{U} \cup \Delta$. If $p_{k}$ is a processor not used in $\delta$, i.e. no task is assigned to it in $\delta$, and $\tau_{i}$ is schedulable on $p_{k}$ for $a$, then $\tau_{i}$ is still schedulable on $p_{k}$ for $a^{\prime}$.

If $\Delta^{*} \neq \emptyset$, because of messages, we cannot conclude about the schedulability of the system without checking the network.

Rule 3 After a decision $\delta$ where $\Delta^{*}=\emptyset$, the domains of non-allocated tasks to check during filtering are reduced to the processor set where a task has been 
added in $\delta$.

\subsection{Reducing further schedulability tests}

Other rules can be introduced to reduce domains during filtering by considering some dominance relationships between tasks. A rule can be deduced from property 11.2 (see Appendix 1):

Rule 4 If, from a schedulable partial allocation, allocating $\tau_{i}$ to $p_{k}$ makes $\tau_{i}$ unschedulable, then $p_{k}$ has to be removed from the domain of all tasks $\tau_{j}$ such that prio $_{j}<$ prio $_{i} \wedge C_{j} \geq C_{i} \wedge T_{j} \leq T_{i}$.

In the same way, the following rule can be stated from property 11.3 (see Appendix 1):

Rule 5 If, from a schedulable partial allocation, allocating $\tau_{i}$ to $p_{k}$ makes an allocated task $\tau_{b}$ unschedulable, then $p_{k}$ has to be removed from the domain of all tasks $\tau_{j}$ with prio $_{j}>$ prio $_{b} \wedge C_{j} \geq C_{i} \wedge T_{j} \leq T_{i}$.

Finally, a trivial propagation rule can be added by considering message schedulability:

Rule 6 If, from a schedulable partial allocation a, allocating a communicating task $\tau_{i}$ makes a message unschedulable, then the domain of $\tau_{i}$ has to become $\cup_{\tau_{j}}\left\{a\left(\tau_{j}\right)\right\}$ where $\tau_{j}$ is an allocated task that exchanges data with $\tau_{i}$.

These rules can be easily implemented in Algorithm 1 on line 8 .

\section{Solving the problem with logic-based Benders decomposition}

Contrary to the global constraint strategy, which includes schedulability in the search algorithm, the approach presented in this section is based on the Benders decomposition and separates resource and allocation constraints from schedulability ones.

\subsection{Benders decomposition scheme}

We only give the basic principles of this technique, for a more detailed description please refer to [12]. Our approach is based on an extension of a Benders scheme. A Benders decomposition [7] is a solving strategy of linear problems 
that uses a partition of the problem among its variables: $x, y$. A master problem considers only $x$, whereas a subproblem tries to complete the assignment on $y$ and produces a Benders cut added to the master. This cut is the central element of the technique, it is usually a linear constraint on $x$ inferred by the dual of the subproblem. Benders decomposition can therefore be seen as a form of learning from mistakes.

For a discrete satisfaction problem, the resolution of the dual consists in computing the unfeasibility proof of the subproblem (in this case, the dual is called an inference dual) and determining under what conditions the proof remains valid to infer valid cuts. The Benders cut can be seen in this context as an explanation of failure that is learnt by the master. We refer here to a more general Benders scheme called logic Benders decomposition [27] where any kind of subproblem can be used as long as the inference dual of the subproblem can be solved.

We propose an approach inspired by methods used to integrate constraint programming into a logic-based Benders decomposition $[61,8]$. The allocation and resource constraints are considered on one side, and timing ones through schedulability on the other (see Fig. 4). The master problem solved with $\mathrm{cp}$ yields a valid allocation. The subproblem checks the schedulability of this allocation, eventually finds out why it is unschedulable and designs a set of constraints, named nogoods, which rules out all the assignments that are unschedulable for the same reason.

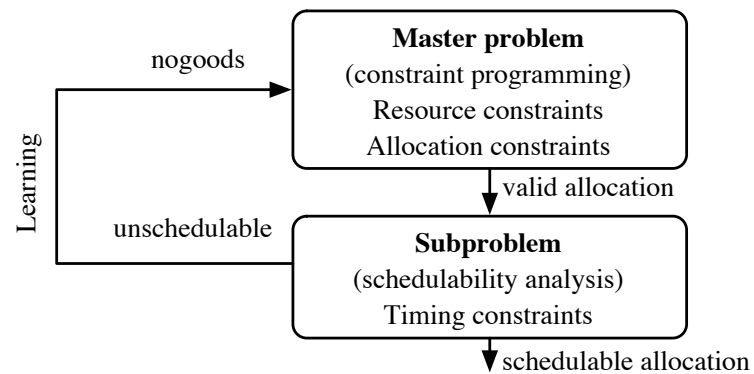

Fig. 4. Logic-based Benders decomposition to solve an allocation problem

\subsection{Cooperation between master and subproblem}

The subproblem considered here is to check whether a valid solution produced by the master problem is schedulable or not. Schedulability analysis is used (see section 2.2.3). We now consider a valid allocation (like the one the $\mathrm{cp}$ solver may propose) in which some tasks are not schedulable. Our purpose is to explain why this allocation is unschedulable, and to translate this into a new constraint for the master problem. 

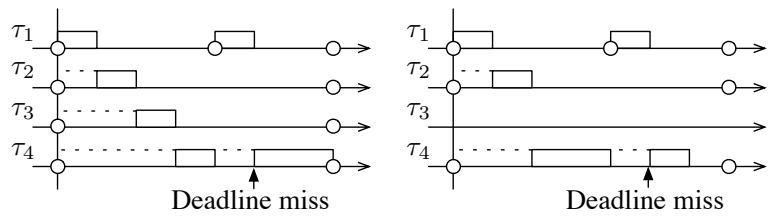

Fig. 5. Illustration of a schedulability analysis. The task $\tau_{4}$ does not meet its deadline. The subset $\left\{\tau_{1}, \tau_{2}, \tau_{4}\right\}$ is identified to explain the unschedulability of the system.

Tasks. The explanation for the unschedulability of a task $\tau_{i}$ is the presence of tasks with higher priority on the same processor that interfere with $\tau_{i}$. For any other allocation with $\tau_{i}$ and $h p_{i}(A)$ on the same processor, it is certain that $\tau_{i}$ will still be detected unschedulable. So, the master problem must be constrained so that all solutions where $\tau_{i}$ and $h p_{i}(A)$ are together are not considered any further. This constraint corresponds to a NotAllEqual ${ }^{6}$ on $x$ :

$$
\operatorname{NotAllEqual}\left(x_{j} \mid \tau_{j} \in S_{i}(A)=h p_{i}(A) \cup\left\{\tau_{i}\right\}\right)
$$

It is worth noticing that this constraint could be expressed as a linear combination of variables $y$. However, $\operatorname{NotAllEqual}\left(x_{1}, x_{3}, x_{4}\right)$ excludes the solutions that contain the tasks $\tau_{1}, \tau_{3}, \tau_{4}$ gathered on any processor.

Nevertheless it is easy to see that this constraint is not totally relevant. For example, in Fig. 5, $\tau_{4}$ that shares a processor with $\tau_{1}, \tau_{2}$ and $\tau_{3}$ misses its deadline. Actually, the set $S_{4}(A)=\left\{\tau_{1}, \tau_{2}, \tau_{3}, \tau_{4}\right\}$ explains the unschedulability but it is not minimal in the sense that, if we remove one task from it, the set is still unschedulable. Here, the set $S_{4}(A)^{\prime}=\left\{\tau_{1}, \tau_{2}, \tau_{4}\right\}$ is sufficient to justify the unschedulability. To explain the unschedulability of a task, there could be more than one minimal task set, this is dependent on the order of enumeration of $h p_{i}(A)$. In the example, $\left\{\tau_{1}, \tau_{3}, \tau_{4}\right\}$ is also a minimal set. However, the more constraints there are, the slower the solver is, thus we consider just one minimal set in explanation.

In order to derive more precise explanations (to achieve a more relevant learning), a conflict detection algorithm, namely QuickXplain [29] (see algorithm 2), has been used to determine a minimal (w.r.t. inclusion) set of involved tasks $S_{i}(A)^{\prime}$. A new function is defined, $R_{i}(X)$, as the worst-case response time of $\tau_{i}$ as if it was scheduled with those tasks belonging to the set $X$ that have priority over it:

$$
R_{i}(X)=C_{i}+\sum_{\tau_{j} \in h p_{i}(A) \cap X}\left\lceil\frac{R_{i}(X)}{T_{j}}\right\rceil C_{j}
$$

\footnotetext{
$\overline{6}$ A NotAllEqual on a set $V$ of variables ensures that at least two variables among $V$ take distinct values.
} 


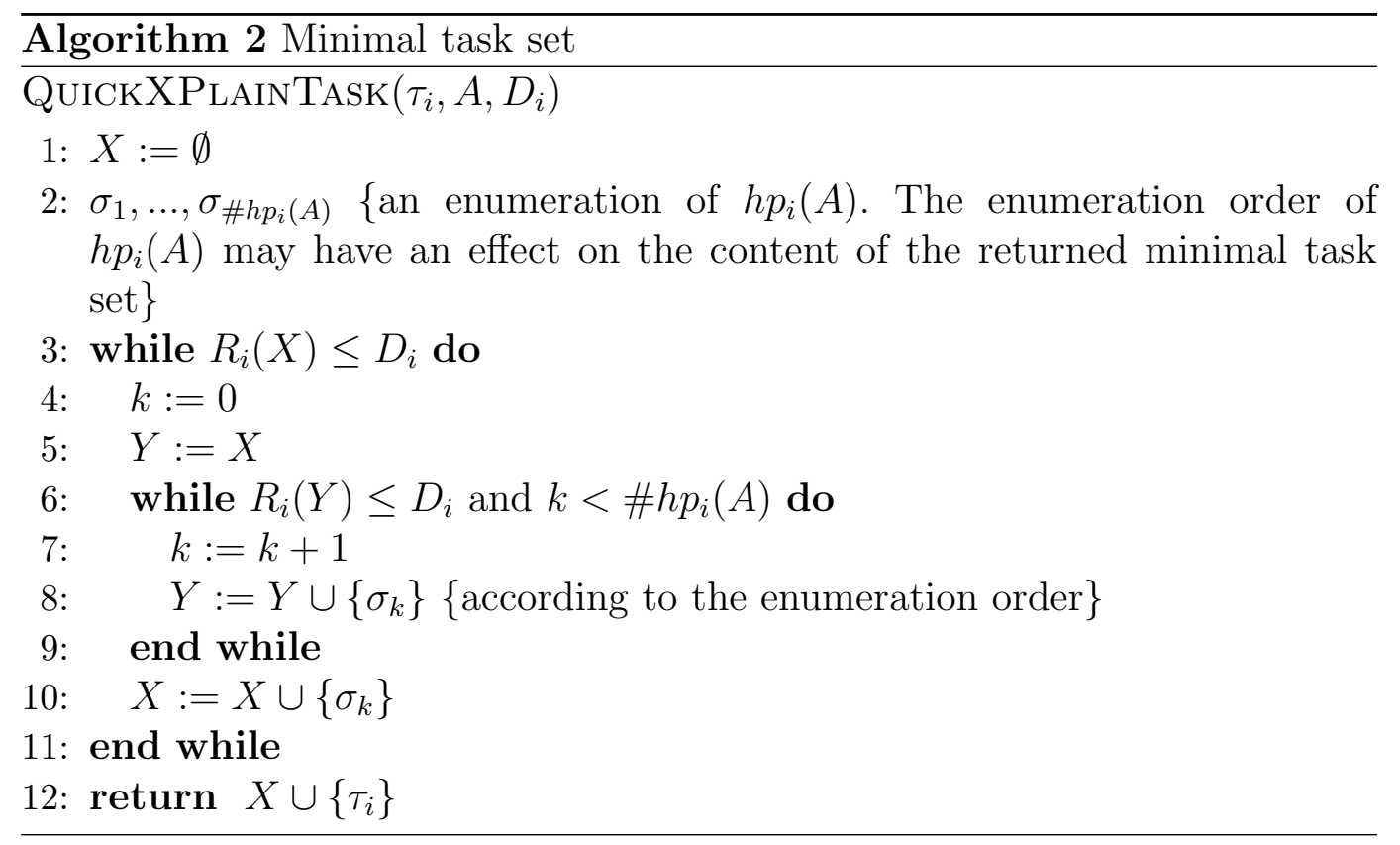

Messages. The reasoning is quite similar. If a message $M_{i j}$ is found unschedulable, it is because of the messages in $h p_{i j}(A)$ and the longest message in $l p_{i j}(A)$. We denote $M_{i j}(A)$ their union together with $\left\{M_{i j}\right\}$. The translation of this information in terms of constraint yields:

$$
\sum_{M_{a b} \in M_{i j}(A)} w_{a b}<\# M_{i j}(A)
$$

It is equivalent to a NotAllEqual constraint on a set of messages since, to be met, it requires that at least one message of $M_{i j}(A)$ "disappears" $\left(w_{a b}=0\right)$.

Like for tasks, so as to reduce the set of involved messages, QUICKXPLAIN has been implemented, using a similar adaptation of Eqs. (9) and (10). It returns a minimal set of messages $M_{i j}(A)^{\prime}$.

\subsection{Applying the method to an example}

An example to illustrate the theory is developed hereafter. It shows how the cooperation between master and subproblems is performed. Table 2 shows the characteristics of the considered hardware architecture (with 4 processors) and Table 3 those of the software architecture (with 20 tasks). The entry $" x, y \rightarrow j$ " for the task $\tau_{i}$ indicates an edge $c_{i j}$ with $C_{i j}=x$ and prio $_{i j}=y$.

The problem is constrained by:

- residence constraints: 
Table 2

\begin{tabular}{|c|c|c|c|c|}
\hline$p_{i}$ & $p_{0}$ & $p_{1}$ & $p_{2}$ & $p_{3}$ \\
\hline$m_{i}$ & 102001 & 280295 & 360241 & 41617 \\
\hline
\end{tabular}

Processor characteristics

Table 3

\begin{tabular}{|c|c|c|c|c|l|}
\hline$\tau_{i}$ & $T_{i}$ & $C_{i}$ & $\mu_{i}$ & prio $_{i}$ & Message \\
\hline$\tau_{0}$ & 36000 & 2190 & 21243 & 1 & $600,1 \rightarrow 13$ \\
$\tau_{1}$ & 2000 & 563 & 5855 & 6 & $500,3 \rightarrow 8$ \\
$\tau_{2}$ & 3000 & 207 & 2152 & 15 & $600,7 \rightarrow 7$ \\
$\tau_{3}$ & 8000 & 2187 & 21213 & 3 & \\
$\tau_{4}$ & 72000 & 17690 & 168055 & 7 & $300,4 \rightarrow 9$ \\
$\tau_{5}$ & 4000 & 667 & 6670 & 8 & $800,5 \rightarrow 19$ \\
$\tau_{6}$ & 12000 & 3662 & 36253 & 14 & \\
$\tau_{7}$ & 3000 & 269 & 2743 & 16 & \\
$\tau_{8}$ & 2000 & 231 & 2263 & 12 & $100,6 \rightarrow 18$ \\
$\tau_{9}$ & 72000 & 6161 & 59761 & 9 & \\
$\tau_{10}$ & 12000 & 846 & 8206 & 4 & $200,2 \rightarrow 15$ \\
$\tau_{11}$ & 36000 & 5836 & 60694 & 20 & \\
$\tau_{12}$ & 9000 & 2103 & 20399 & 10 & \\
$\tau_{13}$ & 36000 & 5535 & 54243 & 13 & \\
$\tau_{14}$ & 18000 & 3905 & 41002 & 18 & \\
$\tau_{15}$ & 12000 & 1412 & 14402 & 5 & \\
$\tau_{16}$ & 6000 & 1416 & 14301 & 17 & $700,8 \rightarrow 17$ \\
$\tau_{17}$ & 6000 & 752 & 7369 & 19 & \\
$\tau_{18}$ & 2000 & 538 & 5487 & 11 & \\
$\tau_{19}$ & 4000 & 1281 & 12425 & 2 & \\
\hline & & & &
\end{tabular}

Task and message characteristics

- $C C_{1}: \tau_{0}$ must be allocated to $p_{0}$ or $p_{1}$ or $p_{2}$.

- $C C_{2}: \tau_{16}$ must be allocated to $p_{1}$ or $p_{2}$.

- $C C_{3}: \tau_{17}$ must be allocated to $p_{0}$ or $p_{3}$.

- co-residence constraints:

- $C C_{4}: \tau_{7}, \tau_{17}$ and $\tau_{19}$ must be on the same processor.

- exclusion constraints:

- $C C_{5}: \tau_{3}, \tau_{11}$ and $\tau_{12}$ must be on different processors.

To start the resolution process, the solver for the master problem finds a valid solution in accordance with $C C_{1}, C C_{2}, C C_{3}, C C_{4}$ and $C C_{5}$. How the cp solver finds such a solution is not our objective here. The valid solution it returns is:

- processor $p_{0}: \tau_{2}, \tau_{5}, \tau_{7}, \tau_{8}, \tau_{9}, \tau_{17}, \tau_{19}$.

- processor $p_{1}: \tau_{4}, \tau_{6}, \tau_{12}, \tau_{13}$.

- processor $p_{2}: \tau_{0}, \tau_{11}, \tau_{14}, \tau_{15}, \tau_{16}$.

- processor $p_{3}: \tau_{1}, \tau_{3}, \tau_{10}, \tau_{18}$.

One deduces that messages are $M_{0,13}, M_{1,8}, M_{4,9}, M_{8,18}, M_{10,15}$, and $M_{16,17}$. 
It is easy to check that it is a valid solution by considering allocation and resource constraints:

- $\mu_{2}+\mu_{5}+\mu_{7}+\mu_{8}+\mu_{9}+\mu_{17}+\mu_{19}=93383 \leq m_{0}$;

- $\mu_{4}+\mu_{6}+\mu_{12}+\mu_{13}=278950 \leq m_{1}$;

- $\mu_{0}+\mu_{11}+\mu_{14}+\mu_{15}+\mu_{16}=151642 \leq m_{2}$;

- $\mu_{1}+\mu_{3}+\mu_{10}+\mu_{18}=40761 \leq m_{3}$;

- $\frac{C_{2}}{T_{2}}+\frac{C_{5}}{T_{5}}+\frac{C_{7}}{T_{7}}+\frac{C_{8}}{T_{8}}+\frac{C_{9}}{T_{9}}+\frac{C_{17}}{T_{17}}+\frac{C_{19}}{T_{19}}=0.972 \leq 1$;

- $\frac{C_{4}}{T_{4}}+\frac{C_{6}}{T_{6}}+\frac{C_{12}}{T_{12}}+\frac{C_{13}}{T_{13}}=0.938 \leq 1$;

- $\frac{C_{0}}{T_{0}}+\frac{C_{11}}{T_{11}}+\frac{C_{14}}{T_{14}}+\frac{C_{15}}{T_{15}}+\frac{C_{16}}{T_{16}}=0.794 \leq 1$;

- $\frac{C_{1}}{T_{1}}+\frac{C_{3}}{T_{3}}+\frac{C_{10}}{T_{10}}+\frac{C_{18}}{T_{18}}=0.894 \leq 1$.

- $\frac{C_{0,13}}{T_{0}}+\frac{C_{1,8}}{T_{1}}+\frac{C_{4,9}}{T_{4}}+\frac{C_{8,18}}{T_{8}}+\frac{C_{10,15}}{T_{10}}+\frac{C_{16,17}}{T_{16}}=0.454 \leq 1$.

The subproblem now checks the schedulability of the valid solution. The schedulability analysis proceeds in three steps.

First step: analyzing the schedulability of tasks. The worst-case response time for each task is obtained by application of Eq. (8) and it is compared with its relative deadline. Here $\tau_{5}, \tau_{12}, \tau_{16}$ and $\tau_{19}$ are found unschedulable.

Second step: analyzing the schedulability of messages. The worst-case response time for each message is obtained by application of Eq. (9) and Eq. (10) and it is compared with its relative deadline. Here $M_{1,8}$ is found unschedulable.

Third step: explaining why this allocation is not schedulable. The unschedulability of $\tau_{5}$ is due to the interference of higher priority tasks on the same processor: $h p_{5}=\left\{\tau_{2}, \tau_{7}, \tau_{8}, \tau_{9}, \tau_{17}\right\}$. By applying QUICKXPLAINTASK (see algorithm 2) with $h p_{5}$ ordered by increasing index, we find $S_{5}(A)^{\prime}=$ $\left\{\tau_{5}, \tau_{9}\right\}$ as the minimal set. Consequently, the explanation of the unschedulability is translated into the new constraint:

$$
C C_{6}: \text { NotAllEqual }\left\{x_{5}, x_{9}\right\}
$$

In the same way, by applying QUICKXPLAINTASK for $\tau_{12}$, we find:

$$
C C_{7}: \text { NotAllEqual }\left\{x_{6}, x_{12}, x_{13}\right\}
$$

for $\tau_{16}$ :

$$
C C_{8}: \text { NotAllEqual }\left\{x_{11}, x_{16}\right\}
$$

and for $\tau_{19}$ :

$$
C_{9}: \operatorname{NotAllEqual}\left\{x_{9}, x_{19}\right\}
$$


For $M_{1,8}$, we have:

$$
M_{1,8}(A)=\left\{M_{0,13}, M_{1,8}, M_{4,9}, M_{8,18}, M_{16,17}\right\} .
$$

QuickXPlain returns $M_{1,8}(A)^{\prime}=\left\{M_{0,13}, M_{1,8}, M_{4,9}, M_{16,17}\right\}$ as the minimal set. So another constraint is created:

$$
C C_{10}: w_{0,13}+w_{1,8}+w_{4,9}+w_{16,17}<4
$$

These new constraints $C C_{6}, C C_{7}, C C_{8}, C C_{9}$ and $C C_{10}$ are added to the master problem. They define a new problem for which it has to search for a valid solution and so on.

After 20 iterations between the master problem and the subproblem, this allocation problem is proven without solution. This results from 78 constraints learnt all along the solving process. This example has been solved using (EDIPE (see Section 8). On a computer with a G4 processor $(800 \mathrm{MHz})$, its computing time was 10.3 seconds.

\section{Experimental results}

We have developed a dedicated tool named CEDIPE [13] that implements our solving approaches. EDIPE is based on the CHOCO [33,31] cp system and PALM [30], an explanation-based cp system.

The time that cp takes to find a solution depends on three parameters: the size of the search space, the ability to reduce that search space, and the way it is searched. In cp the search of solutions is managed by a search strategy that aims at quickly directing the search towards good solutions without loosing the completeness. A search strategy is related to the order of choice of variable and value. It consists in defining an algorithm that specifies at each branching the next variable to be chosen, together with its value. Different approaches have been studied in [23] (conduct by the domain sizes, by the task worst case execution times, etc.) and the most efficient strategy we found is a general search strategy for constraint programming inspired by integer programming techniques and based on the concept of impact of a variable [48]. The impact measures the importance of a variable for the reduction of the search space. Impacts are learnt from the observation of domain reduction during search and need no tuning. Experiments have been conduct with this search strategy.

For the allocation problem, no specific benchmarks are available as a reference in the real-time community. Experiments are usually done on didactic 
examples $[63,4]$ or randomly generated configurations $[47,39]$. We adopted the latter solution. Our generator takes several parameters into account:

- Tasks. The number of tasks, $n$, is fixed at 40. Periods are generated to have a limitation of the hyper-period while various values are possible [22]. Priorities are randomly assigned. Worst-case response times are generated with the routine UUNIFAST [9], which implements an algorithm for efficiently generating unbiased vectors of utilization. A parameter, $\%_{\text {global }}$, is used to scale the global utilization such that $\sum_{i=1}^{n} C_{i} / T_{i}=m \%$ global. The difficulty of a problem related to schedulability is evaluated using \% global, which varies from 40 to $90 \%$.

- Communications. The number of communications, mes, i.e. the number of edges in the task graph, and the network workload, $\%_{m e s}$, impact on the difficulty of a problem. For the sake of simplicity, only linear data communications between tasks are considered and the priority of a message is inherited from the task producing it. Worst-case transmission times are generated with the same technique as worst-case execution times such that $\sum_{i=1}^{n} C_{i j} / T_{i}=\%_{m e s}$.

- Processors. The number of processors, $m$, is fixed at 7 . The memory capacity of a processor is generated such that $\sum_{k=1}^{m} m_{k}=\left(1+\%_{m e m}\right) \sum_{i=1}^{n} \mu_{i}$ where $\%_{\text {mem }}$ represents the global memory over-capacity for the hardware architecture. The memory over-capacity has a significant impact on the difficulty of a problem (a very low capacity can lead to solving a packing problem, sometimes very difficult).

- Allocation constraints. The percentage of tasks involved in allocation constraints is given by the parameters $\%_{r e s}$ for residence constraints, $\%_{c o}$ for co-residence, and $\%_{\text {exc }}$ for exclusion constraints. Different constraints are sized such that $\%_{\text {res }}, \%_{c o}$ and $\%_{\text {exc }}$ are respected. The allocation difficulty for a problem is given by these percentages.

Several classes of problems have been defined depending on the difficulty of both allocation and schedulability problems. Table 4 describes the parameters of each basic difficulty class. By combining them, categories of problems can be specified. For instance, a W-X-Y-Z category corresponds to problems with a memory difficulty in class $\mathrm{W}$, an allocation difficulty in class X, a schedulability difficulty in class $\mathrm{Y}$ and a network difficulty in class $\mathrm{Z}$.

Table 4

\begin{tabular}{|c|c|c|c|c|c|c|c|c|c|c|}
\hline \multicolumn{2}{|c|}{ Memory } & \multicolumn{4}{|c|}{ Allocation } & \multicolumn{2}{|c|}{ Schedulability } & \multicolumn{3}{|c|}{ Message } \\
\hline & $\%_{\text {mem }}$ & & $\%_{\text {res }}$ & $\%_{c o}$ & $\%_{\text {exc }}$ & & $\%_{\text {global }}$ & & mes & $\%_{m e s}$ \\
\hline 1 & 60 & 1 & 0 & 0 & 0 & 1 & 40 & 1 & 0 & 0 \\
\hline 2 & 30 & 2 & 15 & 15 & 15 & 2 & 60 & 2 & 20 & 70 \\
\hline 3 & 10 & 3 & 33 & 33 & 33 & 3 & 90 & 3 & 30 & 150 \\
\hline
\end{tabular}

Details of difficulty classes 


\subsection{Results}

The method with the global constraint is denoted as Global-CPRTA and the Benders decomposition method is denoted by Benders-CPRTA. A problem with a solution is called a consistent problem. A problem for which the proof that no solution exists is given, is called an inconsistent problem. A problem for which we cannot state whether a solution exists or not, is an open problem. A time limit is fixed at 10 minutes per problem. If the problem is still open within this time limit, the search is stopped.

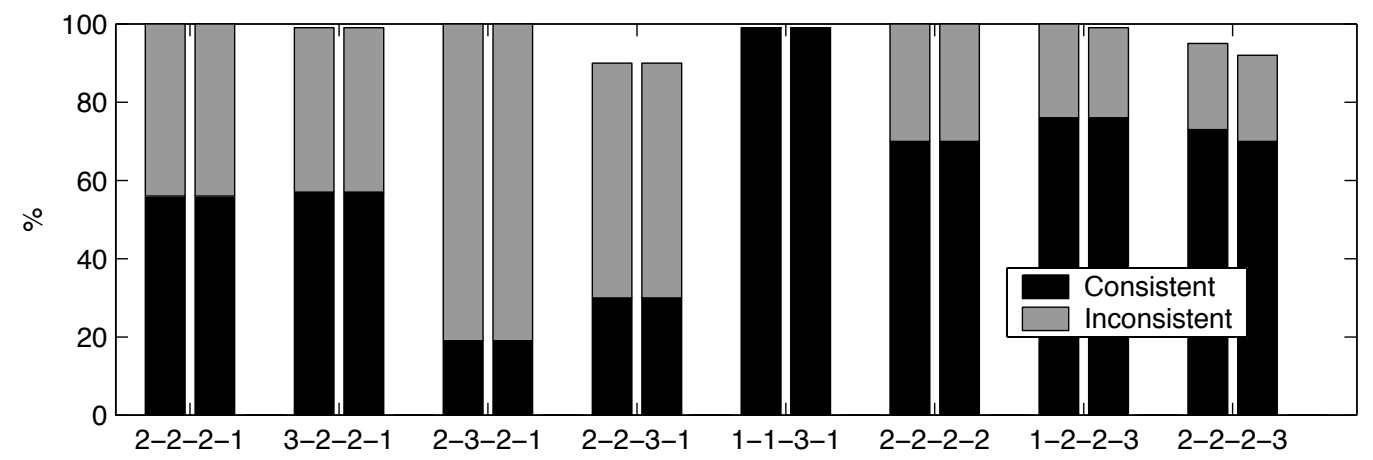

Fig. 6. Sum of percentages (y-axis) of consistent (black) and inconsistent (grey) problems for each difficulty class (x-axis) with Benders-CPRTA (right) and Global-CPRTA (left)

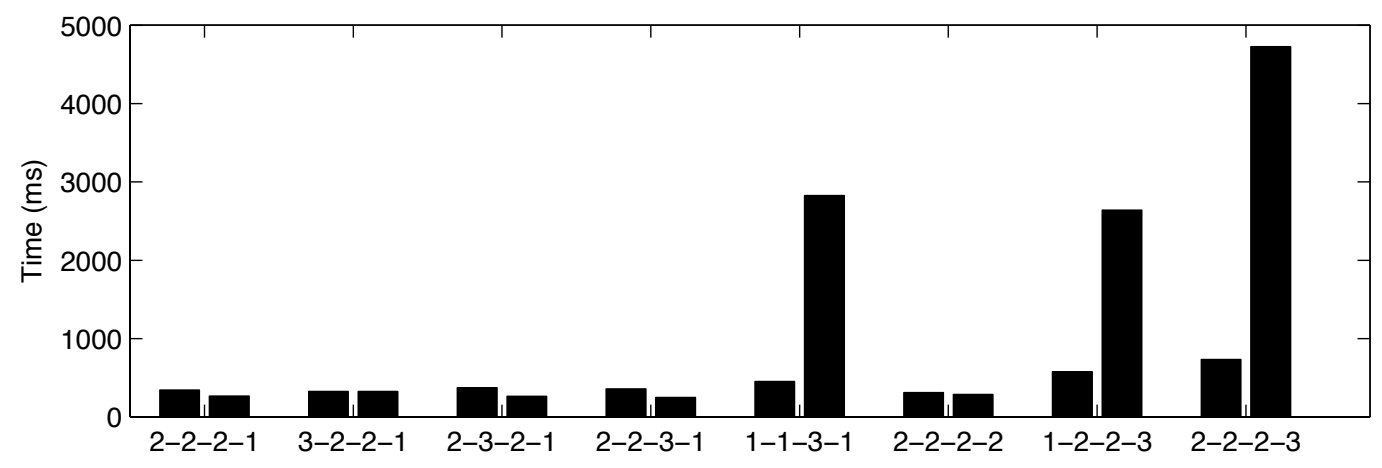

Fig. 7. Median time (y-axis) for solving a problem (consistent and inconsistent) for each difficulty class (x-axis) with Benders-CPRTA (right) and Global-CPRTA (left)

Figures 6 and 7 summarize some of the results (Appendix 2 gives detailed results). We do not give the results for all the intermediate classes of problems (like 1-1-1-1, 2-1-1-1, etc.) because they are easily solved and they do not exhibit a specific behavior. The data are obtained on 100 instances (40 tasks, 7 processors $)$ per class of difficulty with a Pentium $4(3.2 \mathrm{GHz})$.

Figure 6 gives the percentage of consistent and inconsistent problems solved by Benders-CPRTA and Global-CPRTA. For a difficulty class, Global-CPRTA is the left bar and Benders-CPRTA is the right one. Benders- and Global-CPRTA 
are efficient for solving problems with less than $20 \%$ of open problems for each difficulty class (and less than 10\% if we do not consider 2-2-3-1).

Figure 7 gives the median time for solving a problem. For hard schedulabilty problems (e.g. class 1-1-3-1) and for a heavy load network (e.g. classes 1-2-2-3 and 2-2-2-3), Benders-CPRTA is clearly slower than Global-CPRTA. We can explain this because, for Benders-CPRTA, the schedulability constraint set is empty at the beginning of the search. Therefore, all the knowledge dealing with schedulability has to be learnt from the subproblem. Furthermore, learning is only effective when a valid solution is produced by the master problem solver and, as a consequence, it is not really integrated into the cp algorithm. GlobalCPRTA improves performances from this point of view. For a heavy loaded network, Benders-CPRTA has the same difficulty in taking into account the schedulability of messages. Moreover, we have observed that nogoods inferred from message unschedulability are usually "weaker" (the search space cut is smaller) than those inferred from task unschedulability. Learning is thus less efficient for this kind of problem.

However, Benders-CPRTA is on average better in time than Global-CPRTA for classes where schedulability constraints are negligible. In these cases, for Benders-CPRTA, memory and allocation constraints are directly considered by the master problem, which does not consider schedulability, contrary to Global-CPRTA, and thus problems are solved faster.

\subsection{Comparison with simulated annealing}

As stated in Section 3, there are numerous search methods for static allocation problems. However, each technique needs some expertise for optimizing it when a new model is considered. For example, for the branch-and-bound technique, branching and bounding algorithms need to be implemented for each specific allocation problem, and the efficiency of the method depends on the effectiveness of them. Moreover, each technique is sensitive to some parameters. For example, a branch-and-bound is sensitive to the enumeration order of variables. Thus, implementing an efficient solving algorithm (with optimization and smart parameters) is a full problem for each method. Establishing a comparison between different techniques is beyond our present concern.

Thus, we have only implemented and optimized a simulated annealing algorithm (SA) for comparison purposes. The SA algorithm is inspired by [63]. This choice was motivated by similarity with our model and because this work is often referred to allocation problems of hard real-time systems. In [63], the energy function takes into account residence, exclusion and memory constraints as well as task deadline constraints. To be consistent with the CPRTA model, 
the schedulability of messages on the CAN bus and co-residence constraints have been integrated in the same way. All constraints are weighted to make the cost of each one uniform in the objective function. We took great care in its implementation so as to reduce the computation time of this energy function. Moreover, different neighbor functions were experimented as well as different parameters like cooling, number of iterations and initial temperature so as to optimize its behavior. Figures 8 and 9 show only the best results computed by SA for all difficulty classes. When an iteration limit is reached, a new initial solution is produced and a new research is done until a time limit fixed at 10 minutes. If this time limit is reached, the problem is considered as open.

Notice that SA is a heuristic method. As a consequence, in our case, SA can only conclude on consistent problems (Fig. 8). SA is always as or less efficient than Global-CPRTA. Figure 9 shows the median time to solve a consistent problem with SA and CPRTA. Global-CPRTA is always faster than SA. Benders-CPRTA is also faster than SA except for difficult schedulability problems 1-1-3-1. All these results show that cp is an efficient way to mix different constraints without an initial step.

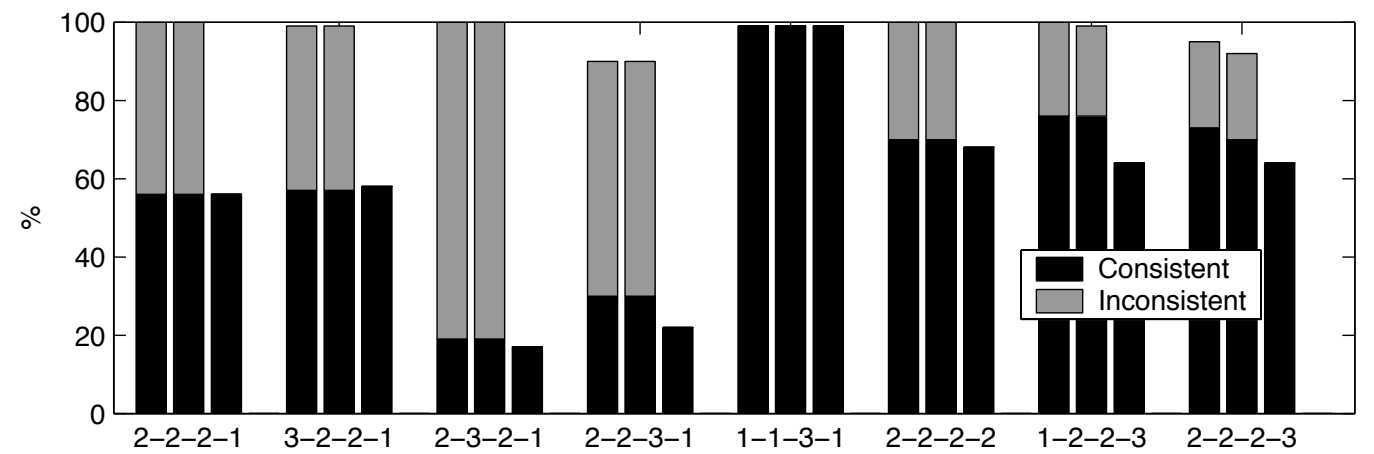

Fig. 8. Sum of percentages (y-axis) of consistent (black) and inconsistent (grey) problems for each difficulty class (x-axis) with SA (right), Benders-CPRTA (middle) and Global-CPRTA (left)

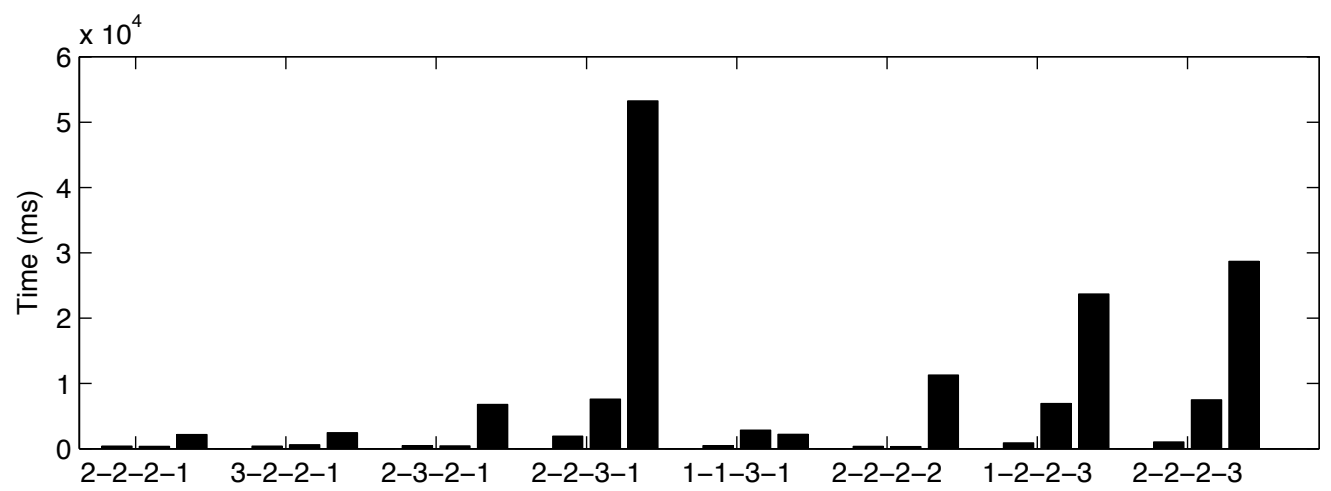

Fig. 9. Median time (y-axis) for solving a problem (consistent and inconsistent) for each difficulty class (x-axis) with SA (right), Benders-CPRTA (middle) and Global-CPRTA (left) 


\section{Explanations}

In comparison with other search methods, using a constraint solver may help "intrinsically" to answer some classical queries when a problem is proved without a solution such as: why does my problem have no solution? Usually, when the domain of a variable of a csp becomes empty (no value exists that will respect all the constraints on that variable), basic cp systems notify the user that there is no solution. Nevertheless, thanks to the versatility of the explanationbased constraint approach we use, those relevant constraints, which explain the failure, are made available [30]. This advantage of the approach is another motivation to go further in the utilization of cp. At present, only BendersCPRTA has been investigated with this objective.

Thus, in the case of an allocation problem for which no solution has been found, we analyze the set of constraints that is returned to explain the problem inconsistency. There can be many reasons to explain inconsistency. At the design level, we would like to be able to incriminate high level characteristics of the system such as: allocation constraints, schedulability requirements of tasks, processors or network limitation. However, two points of view, based on the software or hardware architecture, can be adopted. To begin, we only focus on the characteristics of the software architecture by analyzing how each task is "responsible" for the failure. We give some insight into the way a critical task from the schedulability point of view can be identified. Each failure of the search process due to schedulability is analyzed and transformed into a constraint criterion that encapsulates an accurate reason for this failure. The study of these criteria may lead to the guilty $\operatorname{task}(\mathrm{s})$. The rationale of this evaluation is based on the following remarks:

- The more a task appears within a nogood, the more this task has an impact on the schedulability inconsistency.

- The level of propagation performed by a nogood (either NotAllEqual( $\left.x_{i}\right)$ or $\left.\sum w_{i j}<B\right)$, i.e its impact within the proof, is strongly related to its size (the number of tasks it involves). "Small" NotAllEqual have greater impact.

In its general form, a constraint (learnt from a nogood) is defined by NotAllEqual $\left(x_{i}\right)$ or $\sum w_{i j}<B$ (see Section 7.2). We denote NAE the set of constraints in the NotAllEqual form and SUM the set of constraints in the second form. For a task $\tau_{i}$ a constraint criterion $\mathcal{C}_{i}$ is evaluated:

$$
\mathcal{C}_{i}=\sum_{\substack{c \in \mathrm{NAE} \\ x_{i} \in c}} \frac{1}{\# c}+\sum_{\substack{c \in \mathrm{SUM} \\ \exists j, w_{i j} \in c \vee w_{j i} \in c}} \frac{1}{\# c}
$$

This criterion considers the presence of a task in each constraint and its im- 
pact. The bigger $\mathcal{C}_{i}$ is, the bigger the impact of $\tau_{i}$ is on the inconsistency. By studying tasks with high $\mathcal{C}_{i}$ and understanding why they have such an impact on the inconsistency (e.g. low priority allocation, too large processor utilization), it is possible to change some requirements (e.g. by adapting priorities, or choosing a different version for a task with another period) and so to obtain a solution for the problem.

Table 5

\begin{tabular}{|c|c||c|c||c|c||c|c|}
\hline$\tau_{i}$ & $\mathcal{C}_{i}$ & $\tau_{i}$ & $\mathcal{C}_{i}$ & $\tau_{i}$ & $\mathcal{C}_{i}$ & $\tau_{i}$ & $\mathcal{C}_{i}$ \\
\hline$\tau_{19}$ & 6.33 & $\tau_{13}$ & 4.78 & $\tau_{2}$ & 3.22 & $\tau_{3}$ & 2.53 \\
$\tau_{14}$ & 5.98 & $\tau_{9}$ & 3.95 & $\tau_{1}$ & 2.85 & $\tau_{16}$ & 2.25 \\
$\tau_{11}$ & 5.98 & $\tau_{6}$ & 3.83 & $\tau_{10}$ & 2.77 & $\tau_{18}$ & 1.97 \\
$\tau_{5}$ & 5.42 & $\tau_{7}$ & 3.45 & $\tau_{4}$ & 2.65 & $\tau_{8}$ & 1.73 \\
$\tau_{12}$ & 5.42 & $\tau_{15}$ & 3.32 & $\tau_{17}$ & 2.55 & $\tau_{0}$ & 1.15 \\
\hline
\end{tabular}

Constraint criteria computed on the example of Section 7.3

Table 5 gives $\mathcal{C}_{i}$ computed with (EDIPE in accordance with equation (15) for the inconsistent example of Section 7.3 with EDIPE [13]. Task $\tau_{19}$ has the biggest $\mathcal{C}_{i}$. This task has a low priority together with a high processor utilization $\left(C_{19} / T_{19}=0.32\right)$. By just changing its priority to the highest one, and reusing Benders-CPRTA, we found a solution for this problem.

Notice that this process consists in analyzing the final set of constraints with a heuristic based on the information about nogoods gathered during the search with Benders decomposition. This process can be generalized to memory and allocation constraints by the use of a specific search technique [48]. However, this work is in progress and still needs more study before becoming significant.

\section{Conclusion and future work}

In this paper, we present an original and complete approach (CPRTA) to solve a hard real-time allocation problem with constraint programming. To tackle this problem, two approaches are proposed. For the first one, timing constraints have been conducted to define a global constraint. This method has been optimized to speed up the search by exploiting specific properties of schedulability analysis. For the second one, a decomposition method built on a logic Benders scheme is used. The whole problem is split into a master problem handling allocation and resource constraints and a subproblem for timing constraints. A rich interaction between master and subproblems is performed with the computation of minimal sets of unschedulable tasks and messages. It implements a learning technique in an effort to combine the various issues into a solution that satisfies all the constraints. 
Experimental results show that these two methods produce an efficient way of solving allocation problems. Thanks to the specialized global constraint, the Global-CPRTA achieves a better performance.

Another important specificity of CPRTA is its completeness, i.e. if a problem has no solution, the search algorithm is able to prove it. In future work, our aim is to integrate into the design process an intelligent tool based on CPRTA able to return pertinent explanations justifying the failure.

In the future, we hope to improve the solving method by considering properties of schedulability tests in a constraint expressed by automata [51]. The problem of priorities assignment is another way to investigate by using the optimal algorithm of Audsley [5] in the Benders decomposition or a new global constraint.

\section{Acknowledgements}

Many thanks to Carol Robins for her excellent work in making the document more readable.

\section{References}

[1] T. F. Abdelzaher and K. G. Shin. Period-based partitioning and assignment for large real-time applications. IEEE Transactions on Computers, 49(1):81-87, 2000 .

[2] J. Aguilar and E. Gelenbe. Task assignment and transaction clustering heuristics for distributed systems. Information Sciences, 97(2):199-219, 1997.

[3] S. Ali, J.-K. Kim, H. Siegel, A. Maciejewski, Y. Yu, S. Gundala, S. Gertphol, and V. Prasanna. Greedy heuristic for resource allocation in dynamic distributed real-time heterogeneous computing systems. In Proceedings of the International Conference on Parallel and Distributed Processing Techniques and Applications (PDPTA 2002), volume 29, 2002.

[4] P. Altenbernd and H. Hansson. The slack method: A new method for static allocation of hard real-time tasks. Real-Time Systems, 15(2):103-130, 1998.

[5] N. Audsley, A. Burns, M. Richardson, K. Tindell, and A. J. Wellings. Applying new scheduling theory to static priority pre-emptive scheduling. Software Engineering Journal, pages 284-292, 1993.

[6] L. Baccouche. Un Mécanisme d'Ordonnancement Distribué de Tâches Temps Réel. PhD thesis, Institut National Polytechnique de Grenoble, 1995. 
[7] J. F. Benders. Partitioning procedures for solving mixed-variables programming problems. Numerische Mathematik, 4:238-252, 1962.

[8] T. Benoist, E. Gaudin, and B. Rottembourg. Constraint programming contribution to Benders decomposition: a case study. Lecture Notes in Computer Science, 2470:603-617, 2002.

[9] E. Bini and G. Buttazzo. Measuring the performance of schedulability tests. Real-Time Systems, 30:129-154, 2005.

[10] Bosch. CAN Specification version 2.0, 1991.

[11] A. Burns, M. Nicholson, K. Tindell, and N. Zhang. Allocating and scheduling hard real-time task on a point-to-point distributed system. In Proceedings of the Workshop on Parallel and Distributed Real-Time Systems, pages 11-20, 1993.

[12] H. Cambazard, P.-E. Hladik, A.-M. Déplanche, N. Jussien, and Y. Trinquet. Decomposition and learning for a hard real-time task allocating problem. In Proceedings of the 10th International Conference on Principles and Practice of Constraint Programming (CP 2004), 2004.

[13] H. Cambazard and P.E. Hladik. EDIPE. http://oedipe.rts-software.org/index.php.

[14] M. Coli and P. Palazzari. A new method for optimisation of allocation and scheduling in real-time applications. In Proceedings of the 7th Euromicro Workshop on Real-Time Systems, pages 262-269, 1995.

[15] M. DiNatale and J. A. Stankovic. Applying of simulated annealing methods to real-time scheduling and jitter control. In proceedings of the 16th IEEE Real-Time Systems Symposium (RTSS 1995), 1995.

[16] C. Ekelin. An Optimization Framework for Scheduling of Embedded Real-Time Systems. PhD thesis, Chalmers University of Technology, 2004.

[17] A. A. Elsadek and B. E. Wells. A heuristic model for task allocation in heterogeneous distributed computing systems. The International Journal of Computers and Their Applications, 6(1), 1999.

[18] F. Ercal, J. Ramanujan, and P. Sadayappan. Task allocation on a hyper-cube by recursive bipartitioning. Journal of Parallel and Distributed Computing, 10:35-44, 1990.

[19] E. Ferro, R. Cayssials, and J. Orozco. Tuning the cost function in a genetic/heuristic approach to the hard real-time multitask-multiprocessor assignment problem. In Proceedings of the 3th World Multiconference on Systemics Cybernetics and Informatics, pages 575-577, 1999.

[20] E. Ferro, D. Sanchez, R. Cayssials, and J. Orozco. New scheduling and assignment real time control task with precedence and deadline constraint in distributed control systems, 2000. url:citeseer.ist.psu.edu/405977.html. 
[21] J. Fredriksson, K. Sandstrm, and M. Åkerholm. Optimizing resource usage in component-based real-time systems. In Proceedings of the 8th International Symposium on Component-based Software Engineering (CBSE8), 2005.

[22] J. Goossens and C. Macq. Limitation of the hyper-period in real-time periodic task set generation. In Proceedings of the RTS Embedded System (RTS'01), pages 133-147, 2001.

[23] P.-E. Hladik, H. Cambazard, A.-M. Déplanche, and N. Jussien. Dynamic constraint programming for solving hard real-time allocation problems. Technical Report 7, IRCCyN, 2005.

[24] P.-E. Hladik, H. Cambazard, A.-M. Déplanche, and N. Jussien. How to solve allocation problems with constraint programming. In Proc. of the Work In Progress of the 17th Euromicro, pages 25-28, Palma de Mallorca, Balearic Islands, Spain, July 2005.

[25] P.-E. Hladik and A.-M. Déplanche. Extension au réseau can des problèmes de placement. Technical Report 4, IRCCyN, 2005.

[26] J. Hooker, G. Ottosson, E. Thorsteinsson, and H. Kim. A scheme for unifying optimization and constraint satisfaction methods. Knowledge Engineering Review, 15(1):11-30, 2000.

[27] J. N. Hooker and G. Ottoson. Logic-based Benders decomposition. Mathematical Programming, 96:33-60, 2003.

[28] V. Jain and I. E. Grossmann. Algorithms for hybrid milp/cp models for a class of optimization problems. INFORMS Journal on Computing, 13:258-276, 2001.

[29] U. Junker. Quickxplain: Conflict detection for arbitrary constraint propagation algorithms. In Proc. of the 8th International Joint Conference on Artificial Intelligence (IJCAI 01), 2001.

[30] Narendra Jussien. The versatility of using explanations within constraint programming. Habilitation thesis of Université de Nantes, 2003.

[31] Cносо. http://choco.sourceforge.net/.

[32] N. Koziris, M. Romesis, P. Tsanakas, and G. Papakonstantinou. An efficient algorithm for the physical mapping of clustered task graphs onto multiprocessor architectures. In Proceedings of the 8th EuroPDP, pages 406-413, 2000.

[33] F. Laburthe. Choco: implementing a cp kernel. In Proceedings of $C P$ 00 Post Conference Workshop on Techniques for Implementing Constraint Programming Systems, 2000.

[34] E. Lawler. Recent results in the theory of machine scheduling. Mathematical Programming: The State of the Art, 1983.

[35] J. P. Lehoczky. Fixed priority scheduling of periodic task sets with arbitrary deadlines. In Proceedings of the 11th IEEE Real-Time Systems Symposium (RTSS 1990), pages 201-209, 1990. 
[36] R. Lepère and D. Trystram. A new clustering algorithm for scheduling task graphs with large communication delays. In Proceedings of the 16th International Parallel and Distributed Processing Symposium (IPDPS 2002, 2002 .

[37] J. Y.-T. Leung and J. Whitehead. On the complexity of fixed-priority scheduling of periodic, real-time tasks. Performance Evaluation, 22:237-250, 1982.

[38] V. Lo. Heuristic algorithms for task assignment in distributed systems. IEEE Transactions on computers, 37(11):1384-1397, 1988.

[39] Y. Monnier, J.-P. Beauvais, and A.-M. Déplanche. A genetic algorithm for scheduling tasks in a real-time distributed system. In Proceedings of the 24th Euromicro Conference, 1998.

[40] T. Muntean and E-G. Talbi. Hill-climbing, simulated annealing and genetic algorithms, a comparative study. In Proceedings of the 26th Hawaii International Conference on Task Scheduling in Parallel and Distributed Systems (HICSS-26), 1993.

[41] M. Mutka and J-P. Li. A tool for allocating periodic real-time tasks to a set of processors. The Journal of Systems and Software, 29(2):135-164, 1995.

[42] J. Oh, H. Bahn, C. Wu, and K. Koh. Pareto-based soft real-time task scheduling in multiprocessor systems. In Proceedings of the Seventh Asia-Pacific Software Engineering Conference (APSEC'00), pages 24-28, 2000.

[43] OSEK Group. OSEK/VDX Communication version 3.0.2.

[44] H-J. Park and B. Kim. An optimal scheduling algorithm for minimizing the computing period of cyclic synchronous tasks on multiprocessors. The Journal of Systems and Software, 56:213-229, 2001.

[45] D-T. Peng, K. Shin, and T. Abdelzaher. Assignment and scheduling communicating periodic tasks in distributed real-time systems. IEEE Transactions on Software Engineering, 23(12), 1997.

[46] S. C. S. Porto and C.C. Ribeiro. A tabu search approach to task scheduling on heterogeneous processors under precedence constraints. International Journal of High-Speed Computing, 7(2), 1993.

[47] K. Ramamritham. Allocation and scheduling of complex periodic tasks. In Proceedings of the 10th International Conference on Distributed Computing Systems (ICDCS 1990), 1990.

[48] P. Refalo. Impact-based search strategies for constraint programming. In Proceedings of the 10th International Conference on Principles and Practice of Constraint Programming (CP 2004), 2004.

[49] J.C. Régin. Constraints and Integer Programming Combined, chapter Global Constraints and Filtering Algorithms. Kluwer, 2003. 
[50] M. Richard, P. Richard, and F. Cottet. Allocating and scheduling tasks in multiple fieldbus real-time systems. In Proceedings of the IEEE Conference on Emerging Technologies and Factory Automation (ETFA), volume 16, pages 137-144, 2003.

[51] G. Richaud, H. Cambazard, B. O'Sullivan, and N. Jussien. Automata for nogood recording in constraint satisfaction problems. In CP06 Workshop on the Integration of SAT and CP techniques, Nantes, France, Sep 2006.

[52] F. Rossi, P. van Beek, and T. Walsh, editors. Handbook of Constraint Programming. Elsevier, 2006.

[53] F. Sandnes. A hybrid genetic algorithm applied to automatic parallel controller code generation. In Proceedings of the 8th Euromicro Workshop on Real-Time Systems, pages 70-75, 1996.

[54] J. Santos, E. Ferro, J. Orozco, and R. Cassials. A heuristic approach to multitask-multiprocessing assignment problem using the empty-slots method and rate monotonic scheduling. Real-Time Systems, 13(2):167-199, 1997.

[55] K. Schild and J. Würtz. Scheduling of time-triggered real-time systems. Constraints, 5(4):335-357, 2000.

[56] M. Silva, C. Cardeira, and Z. Mammeri. Solving real-time scheduling problems with hopfield-type neural networks. In Proceedings of the Euromicro Conference, pages 671-678, 1997.

[57] H. Stone. Multiprocessor scheduling the aid of network flow algorithms. IEEE Transactions on Software Engineering, 3(1):85-93, 1977.

[58] R. Szymanek, F. Gruian, and K. Kuchcinski. Digital systems design using constraint logic programming. In Proceedings of The Practical Application of Constraint Technologies and Logic Programming (PACLP 2000), 2000.

[59] R. Szymanek and K. Kuchcinski. Partial task assignment of task graphs under heterogeneous resource constraints. In Proceedings of the 40 th conference on Design Automation (DAC '03), pages 244-249, 2003.

[60] E-G. Talbi and T. Muntean. General heuristics for the mapping problem. In Proceedings of the World Transputer Conference, 1993.

[61] E. S. Thorsteinsson. Branch-and-check: A hybrid framework integrating mixed integer programming and constraint logic programming. Lecture notes in Computer Science, 2239, 2001.

[62] K. Tindell, H. Hansson, and A. Wellings. Analysis real-time communications: controller area network (CAN). In Proceedings of the 15th IEEE Real-Time Systems Symposium (RTSS 1994), pages 259-265, 1994.

[63] K. W. Tindell, A. Burns, and A. Wellings. Allocating hard real-time tasks: An np-hard problem made easy. Real-Time Systems, 4(2):145-165, 1992. 
[64] L. Vargas and R. Olivaira. Empirical study of tabu search, simulated annealing and multi-start in fieldbus scheduling. In Proceedings of the 10th IEEE International Conference on Emerging Technologies and Factory Automation (ETFA 2005), volume 1, pages 101-108, 2005.

[65] S. Wang, J. Merrick, and K. Shin. Component allocation with multiple resource constraints for large embedded real-time software design. In Proceedings of the 10th IEEE Real-Time and Embedded Technology and Applications Symposium (RTAS'04), 2004.

[66] C. Wong, F. Thoen, K. Catthoor, and D. Verkest. Requirements for static task scheduling in real-time embedded systems. In Proceedings of the 3rd Workshop on System Design Automation (SDA 2000), pages 23-30, 2000.

\section{Appendix 1: Properties of the schedulability analysis}

Property 11.1 If a partial allocation a is unschedulable, then all decisions from a produce an unschedulable allocation.

Proof: Consider Eq. 8 that defines $R_{i}(a)$, its computation is derived by iteratively calculating formula $R_{i}^{(n)}(a)=C_{i}+\sum_{\tau_{j} \in h p_{i}(a)}\left\lceil\frac{R_{i}^{(n-1)}(a)}{T_{j}}\right\rceil C_{j}$ with $R_{i}^{(0)}(a)=$ $C_{i}+\sum_{\tau_{j} \in h p_{i}(a)} C_{j}$

Property is proved if for a task $\tau_{i} \in \mathcal{U}$, all decisions $\delta$ from $a$ are such that $R_{i}\left(a^{\prime}\right)>T_{i}$ with $a^{\prime}=a+\delta$. It is easy to prove it by induction by pointing out that $h p_{i}(a) \subset h p_{i}\left(a^{\prime}\right)$.

Induction basis. By definition $R_{i}^{(0)}\left(a^{\prime}\right) \geqslant R_{i}^{(0)}(a)$.

Induction step. Assume that $R_{i}^{(n)}\left(a^{\prime}\right) \geqslant R_{i}^{(n)}(a)$ is true. Therefore,

$$
\begin{aligned}
R_{i}^{(n+1)}\left(a^{\prime}\right) & \geqslant C_{i}+\sum_{\tau_{j} \in h p_{i}\left(a^{\prime}\right)}\left\lceil\frac{R_{i}^{(n)}(a)}{T_{j}}\right\rceil C_{j} \\
& =C_{i}+\sum_{\tau_{j} \in h p_{i}(a)}\left\lceil\frac{R_{i}^{(n)}(a)}{T_{j}}\right\rceil C_{j}+\sum_{\tau_{j} \in h p_{i}(\delta)}\left\lceil\frac{R_{i}^{(n)}(a)}{T_{j}}\right\rceil C_{j} \\
& \geqslant R_{i}^{(n+1)}(a)
\end{aligned}
$$

At the fix-point, we have : $R_{i}\left(a^{\prime}\right) \geqslant R_{i}(a)>T_{i}$.

The same reasoning could be made for messages by considering Eq.11 and Eq.12. For a messsage $M_{i j}$ we have $h p_{i j}(a) \subset h p_{i j}\left(a^{\prime}\right)$ and $l p_{i j}(a) \subset l p_{i j}\left(a^{\prime}\right)$ 
Property 11.2 Consider a schedulable partial allocation $a$ and $\delta$, a decision from a that allocates only $\tau_{i}$ with $\delta\left(\tau_{i}\right)=p_{k}$. If $\tau_{i}$ is unschedulable in $a^{\prime}=a+\delta$, then all decisions from a that allocate a task $\tau_{j}$ with prio prio $_{j} \wedge \mathrm{prio}_{j} \geq$ $C_{i} \wedge T_{j} \leq T_{i}$ on $p_{k}$ produce unschedulable allocations.

Proof: Consider a partial schedulable allocation $a$ and two others $a^{\prime}$ and $a^{\prime \prime}$ such that $a^{\prime}=a+\delta_{1}, a^{\prime \prime}=a+\delta_{2}, \delta_{1}\left(\tau_{i}\right)=\delta_{2}\left(\tau_{j}\right)=p_{k}, R_{i}\left(a^{\prime}\right)>T_{i}$ and prio $_{j}<$ prio $_{i} \wedge C_{j} \geq C_{i} \wedge T_{j} \leq T_{i}$. We want to prove that $R_{j}\left(a^{\prime \prime}\right)>T_{j}$.

By definition of priorities, $h p_{j}\left(a^{\prime \prime}\right) \supseteq h p_{i}\left(a^{\prime}\right)$.

The property will be proved by induction.

Induction basis. $R_{i}^{(0)}\left(a^{\prime}\right)=C_{i}+\sum_{\tau_{x} \in h p_{i}\left(a^{\prime}\right)} C_{x} \leqslant C_{j}+\sum_{\tau \in h p_{j}\left(a^{\prime \prime}\right)} C=R_{j}^{(0)}\left(a^{\prime \prime}\right)$.

Induction step. Assume that $R_{j}^{(n)}\left(a^{\prime \prime}\right) \geqslant R_{i}^{(n)}\left(a^{\prime}\right)$ is true. Therefore,

$$
\begin{aligned}
R_{j}^{(n+1)}\left(a^{\prime \prime}\right) & \geqslant C_{i}+\sum_{\tau_{x} \in h p_{j}\left(a^{\prime \prime}\right)}\left\lceil\frac{R_{i}^{(n)}\left(a^{\prime}\right)}{T}\right\rceil C_{x} \\
& =C_{i}+\sum_{\substack{\tau_{x} \in h p_{i}\left(a^{\prime}\right) \\
T}}\left\lceil\frac{R_{i}^{(n)}\left(a^{\prime}\right)}{T}\right\rceil C_{x}+\sum_{\tau_{x} \in h p_{j}\left(a^{\prime \prime}\right)-h p_{i}\left(a^{\prime}\right)}\left\lceil\frac{R_{i}^{(n)}\left(a^{\prime}\right)}{T}\right\rceil C_{x} \\
& \geqslant R_{i}^{(n+1)}\left(a^{\prime}\right)
\end{aligned}
$$

At the fix-point, we have $: R_{j}\left(a^{\prime \prime}\right) \geqslant R_{i}\left(a^{\prime}\right)>T_{i} \geqslant T_{j}$

Property 11.3 Consider a schedulable partial allocation a and $\delta$, a decision from a that allocates only $\tau_{i}$ with $\delta\left(\tau_{i}\right)=p_{k}$ and where $\tau_{b}$ is unschedulable due to $\tau_{i}$, then all decisions from a that allocate a task $\tau_{j}$ with prio pro $_{j}>$ prio $_{b} \wedge C_{j} \geq$ $C_{i} \wedge T_{j} \leq T_{i}$ on $p_{k}$ produce unschedulable allocations.

Proof: Consider a partial allocation $a$, two others $a^{\prime}$ and $a^{\prime \prime}$ such that $a^{\prime}=$ $a+\delta_{1}, a^{\prime \prime}=a+\delta_{2}, \delta_{1}\left(\tau_{i}\right)=\delta_{2}\left(\tau_{j}\right)=\delta_{1}\left(\tau_{b}\right)=\delta_{2}\left(\tau_{b}\right)=p_{k}, R_{b}\left(a^{\prime}\right)>T_{b}$ and prio $_{j}>$ prio $_{b} \wedge C_{j} \geq C_{i} \wedge T_{j} \leq T_{i}$. We want to prove that $R_{b}\left(a^{\prime \prime}\right)>T_{b}$.

By definition of priorities, $h p_{b}\left(a^{\prime \prime}\right)-\left\{\tau_{j}\right\}=h p_{b}\left(a^{\prime}\right)-\left\{\tau_{i}\right\}=h p_{b}$.

The property will be proved by induction.

Induction basis. $R_{b}^{(0)}\left(a^{\prime}\right)=C_{b}+\sum_{\tau \in h p_{b}} C_{j}+C_{i} \leqslant C_{b}+\sum_{\tau \in h p_{b}} C+C_{j}=$ $R_{b}^{(0)}\left(a^{\prime \prime}\right)$.

Induction step. Assume that $R_{b}^{(n)}\left(a^{\prime \prime}\right) \geqslant R_{b}^{(n)}\left(a^{\prime}\right)$ is true. Therefore, 


$$
\begin{aligned}
R_{b}^{(n+1)}\left(a^{\prime \prime}\right) & \geqslant C_{b}+\sum_{\tau \in h p_{b}}\left\lceil\frac{R_{b}^{(n)}\left(a^{\prime}\right)}{T}\right\rceil C+\left\lceil\frac{R_{b}^{(n)}\left(a^{\prime}\right)}{T_{i}}\right\rceil C_{i} \\
& \geqslant R_{b}^{(n+1)}\left(a^{\prime}\right)
\end{aligned}
$$

At the fix-point, we have : $R_{b}\left(a^{\prime \prime}\right) \geqslant R_{b}\left(a^{\prime}\right)>T_{b}$

\section{Appendix 2: Experimental results}

The following tables are the detailed forms of the results shown in Section 8 . $\mathrm{CPU}$ is the computation time in milli-seconds. NODE is the number of nodes explored during the search with the Global-CPRTA. ITER is the number of iterations between the master problem and the subproblem for the BendersCPRTA. NOG is the mean of nogoods inferred from the subproblem for the Benders-CPRTA. The data are obtained on 100 instances (40 tasks, 7 processors) per class of difficulty with a Pentium 4 (3.2 GHz).

\begin{tabular}{|c|c|c|c|c|c|c|}
\hline & CPU & NODE & CPU & NODE & $\mathrm{CPU}$ & NODE \\
\hline $2-2-2-1$ & \multicolumn{2}{|c|}{100 solved } & \multicolumn{2}{|c|}{56 consistent } & \multicolumn{2}{|c|}{44 inconsistent } \\
\hline Median & 343.0 & 32.0 & 359.5 & 35.0 & 297.0 & 1.0 \\
\hline $3-2-2-1$ & \multicolumn{2}{|c|}{99 solved } & \multicolumn{2}{|c|}{57 consistent } & \multicolumn{2}{|c|}{42 inconsistent } \\
\hline Median & 328.0 & 30.0 & 359.0 & 34.0 & 296.5 & 1.0 \\
\hline $2-3-2-1$ & \multicolumn{2}{|c|}{100 solved } & \multicolumn{2}{|c|}{19 consistent } & \multicolumn{2}{|c|}{81 inconsistent } \\
\hline Median & 375.0 & 1.0 & 437.0 & 25.0 & 344.0 & 1.0 \\
\hline $2-2-3-1$ & \multicolumn{2}{|c|}{90 solved } & \multicolumn{2}{|c|}{30 consistent } & \multicolumn{2}{|c|}{60 inconsistent } \\
\hline Med & 59.0 & 1.0 & 1906 & 604 & 328.0 & 1.0 \\
\hline $1-1-3-1$ & \multicolumn{2}{|c|}{99 solved } & \multicolumn{2}{|c|}{99 consistent } & \multicolumn{2}{|c|}{0 inconsistent } \\
\hline Median & 453.0 & 78.0 & 453.0 & 78.0 & 0.0 & 0.0 \\
\hline $2-2-2-2$ & \multicolumn{2}{|c|}{100 solved } & \multicolumn{2}{|c|}{70 consistent } & \multicolumn{2}{|c|}{30 inconsistent } \\
\hline Median & 312.0 & 30.0 & 328.0 & 33.0 & 266.0 & 1.0 \\
\hline $1-2-2-3$ & \multicolumn{2}{|c|}{100 solved } & \multicolumn{2}{|c|}{76 consistent } & \multicolumn{2}{|c|}{24 inconsistent } \\
\hline Median & 578.0 & 72.0 & 851.5 & 160.0 & 297.0 & 1.0 \\
\hline $2-2-2-3$ & \multicolumn{2}{|c|}{95 solved } & \multicolumn{2}{|c|}{73 consistent } & \multicolumn{2}{|c|}{22 inconsistent } \\
\hline Median & 734.0 & 120.0 & 1016.0 & 166.0 & 289.5 & 1.0 \\
\hline
\end{tabular}

Table 6

Results for Global-CPRTA 


\begin{tabular}{|c|c|c|c|c|c|c|c|c|c|}
\hline & $\mathrm{CPU}$ & ITER & NOG & $\mathrm{CPU}$ & ITER & NOG & $\mathrm{CPU}$ & ITER & NOG \\
\hline $2-2-2-1$ & \multicolumn{3}{|c|}{100 solved } & \multicolumn{3}{|c|}{56 consistent } & \multicolumn{3}{|c|}{44 inconsistent } \\
\hline Median & 266.0 & 12.0 & 44.0 & 343.5 & 18.5 & 83.5 & 203.0 & 1.0 & 13.0 \\
\hline $3-2-2-1$ & \multicolumn{3}{|c|}{99 solved } & \multicolumn{3}{|c|}{57 consistent } & \multicolumn{3}{|c|}{42 inconsistent } \\
\hline Median & 328.0 & 20.0 & 61.0 & 594.0 & 42.0 & 147.0 & 219.0 & 1.0 & 11.0 \\
\hline $2-3-2-1$ & \multicolumn{3}{|c|}{100 solved } & \multicolumn{3}{|c|}{19 consistent } & \multicolumn{3}{|c|}{81 inconsistent } \\
\hline Median & 265.0 & 1.0 & 14.0 & 422.0 & 29.0 & 111.0 & 250.0 & 1.0 & 13.0 \\
\hline $2-2-3-1$ & \multicolumn{3}{|c|}{90 solved } & \multicolumn{3}{|c|}{30 consistent } & \multicolumn{3}{|c|}{60 inconsistent } \\
\hline Median & 250.0 & 2.0 & 22.5 & 7586.0 & 80.0 & 383.0 & 219.0 & 1.0 & 15.5 \\
\hline $1-1-3-1$ & \multicolumn{3}{|c|}{99 solved } & \multicolumn{3}{|c|}{99 consistent } & \multicolumn{3}{|c|}{0 inconsistent } \\
\hline Median & 2828.0 & 78.0 & 367.0 & 2828.0 & 78.0 & 367.0 & 0.0 & 0.0 & 0.0 \\
\hline $2-2-2-2$ & \multicolumn{3}{|c|}{100 solved } & \multicolumn{3}{|c|}{70 consistent } & \multicolumn{3}{|c|}{30 inconsistent } \\
\hline Median & 289.5 & 12.0 & 40.0 & 313.0 & 18.0 & 60.0 & 226.5 & 1.0 & 9.5 \\
\hline $1-2-2-3$ & \multicolumn{3}{|c|}{99 solved } & \multicolumn{3}{|c|}{76 consistent } & \multicolumn{3}{|c|}{23 inconsistent } \\
\hline Median & 2641.0 & 152.0 & 375.0 & 6891.0 & 302.5 & 938.0 & 281.0 & 1.0 & 15.0 \\
\hline $2-2-2-3$ & \multicolumn{3}{|c|}{92 solved } & \multicolumn{3}{|c|}{70 consistent } & \multicolumn{3}{|c|}{22 inconsistent } \\
\hline Median & 4726.5 & 179.5 & 452.5 & 7476.5 & 289.0 & 859.5 & 265.0 & 1.0 & 11.5 \\
\hline
\end{tabular}

Table 7

Results for Benders-CPRTA

\begin{tabular}{|c|c|c|c|c|c|c|c|c|}
\hline Class & $\mathbf{2 - 2 - 2 - 1}$ & $\mathbf{3 - 2 - 2 - 1}$ & $\mathbf{2 - 3 - 2 - 1}$ & $\mathbf{2 - 2 - 3 - 1}$ & $\mathbf{1 - 1 - 3 - 1}$ & $\mathbf{2 - 2 - 2 - 2}$ & $\mathbf{1 - 2 - 2 - 3}$ & $\mathbf{2 - 2 - 2 - 3}$ \\
\hline \% consistent & 56.0 & 58.0 & 17.0 & 22.0 & 99.0 & 68.0 & 64.0 & 64.0 \\
\hline Median time & 2140.0 & 2438.0 & 6750.0 & 53234.5 & 2187.0 & 11273.0 & 23664.0 & 28664.0 \\
\hline
\end{tabular}

Table 8

Results for SA 